\title{
From Task Tuning to Task Assignment in Privacy-Preserving Crowdsourcing Platforms
}

\author{
Joris Duguépéroux, Tristan Allard \\ Univ Rennes, CNRS, IRISA \\ Rennes, France \\ \{firstname.name\}@irisa.fr
}

\begin{abstract}
Specialized worker profiles of crowdsourcing platforms may contain a large amount of identifying and possibly sensitive personal information (e.g., personal preferences, skills, available slots, available devices) raising strong privacy concerns. This led to the design of privacypreserving crowdsourcing platforms, that aim at enabling efficient crowdsourcing processes while providing strong privacy guarantees even when the platform is not fully trusted. In this paper, we propose two contributions. First, we propose the PKD algorithm with the goal of supporting a large variety of aggregate usages of worker profiles within a privacypreserving crowdsourcing platform. The PKD algorithm combines together homomorphic encryption and differential privacy for computing (perturbed) partitions of the multi-dimensional space of skills of the actual population of workers and a (perturbed) COUNT of workers per partition. Second, we propose to benefit from recent progresses in Private Information Retrieval techniques in order to design a solution to task assignment that is both private and affordable. We perform an in-depth study of the problem of using PIR techniques for proposing tasks to workers, show that it is NP-Hard, and come up with the PKD PIR Packing heuristic that groups tasks together according to the partitioning output by the PKD algorithm. In a nutshell, we design the PKD algorithm and the PKD PIR Packing heuristic, we prove formally their security against honest-butcurious workers and/or platform, we analyze their complexities, and we demonstrate their quality and affordability in real-life scenarios through an extensive experimental evaluation performed over both synthetic and realistic datasets.
\end{abstract}

\section{Introduction}

Crowdsourcing platforms are online intermediates between requesters and workers. The former have tasks to propose to the latter, while the latter have profiles (e.g., skills, devices, experience, availabilities) to propose to the former. Crowdsourcing platforms have grown in diversity, covering application domains ranging 
from micro-task $\mathrm{P}^{1}$ or home-cleaning ${ }^{2}$ to collaborative engineering ${ }^{3}$ or specialized software team design 4 .

The efficiency of crowdsourcing platforms especially relies on the wealth of information available in the profiles of registered workers. Depending on the platform, a profile may indeed contain an arbitrary amount of information: professional or personal skills, daily availabilities, minimum wages, diplomas, professional experiences, centers of interest and personal preferences, devices owned and available, etc. This holds especially for platforms dedicated to specialized tasks that require strongly qualified worker: 5 . But even micro-tasks platforms may maintain detailed worker profiles (see, e.g., the qualification system of Amazon Mechanical Turk that maintains so-called premium qualifications ${ }^{6}$ - i.e., sociodemographic information such as age range, gender, employment, marital status, etc. - in the profiles of workers willing to participate to surveys). The availability of such detailed worker profiles is of utmost importance to both requesters and platforms because it enables:

Primary usages of worker profiles: to target the specific set of workers relevant for a given task (through e.g., elaborate task assignment algorithms [29]).

Secondary usages of worker profiles: to describe the population of workers available, often through COUNT aggregates, in order e.g., to promote the platform by ensuring requesters that workers relevant for their tasks are registered on the platform 7 , or to participate to the task design by letting requesters fine-tune the tasks according to the actual population of workers (e.g., setting wages according to the rarity of the skills required, adapting slightly the requirements according to the skills available).

Both primary and secondary usages are complementary and usually supported by today's crowdsourcing platforms, in particular by platforms dedicated to highly skilled tasks and worker: 8

However, the downside of fine-grained worker profiles is that detailed information related to personal skills can be highly identifying (e.g., typically a unique combination of location/skills/centers of interest) or sensitive (e.g., costly devices or high minimum wages may correspond to a wealthy individual, various

1 https://www.mturk.com/

2 https://www.handy.com/

3 https://www.kicklox.com/

4 https://tara.ai/

${ }^{5}$ We adopt in this paper a broad definition of crowdsourcing, including in particular freelancing platforms (similarly to [28]).

6 https://requester.mturk.com/pricing

7 See for example the Kicklox search form (https://www.kicklox.com/en/) that inputs a list of keywords (typically skills) and displays the corresponding number of workers available.

8 See for example, Kicklox (https://www.kicklox.com/en/) or Tara (https://tara.ai/). The secondary usage consisting in promoting the platform is sometimes performed through a public access to detailed parts of worker profiles (e.g., Malt (https: //www.malt.com/), 404works (https://www.404works.com/en/freelancers)). 
personal traits may be inferred from centers of interest $t^{9}$. Recent privacy scandals have shown that crowdsourcing platforms are not immune to negligences or misbehaviours. Well-known examples include cases where personally identifiable information of workers is trivially exposed online 26] or where precise geolocations are illegitimately accessed 10 .11. It is noticeable that workers nevertheless expect platforms to secure their data and to protect their privacy in order to lower the privacy threats they face [39]. Moreover, in a legal context where laws firmly require businesses and public organizations to safeguard the privacy of individuals (such as the European GDPR ${ }^{12}$ or the California Consumer Privacy Act ${ }^{13}$ ), legal compliance is also a strong incentive for platforms for designing and implementing sound privacy-preserving crowdsourcing processes. Ethics in general, and privacy in particular, are indeed clearly identified as key issues for next generation future of work platforms [32]. Most related privacy-preserving works have focused on the primary usage of worker profiles, i.e., the task-assignment problem (e.g., based on additively-homomorphic encryption [20] or on local differential privacy [5] $\left.{ }^{14}\right]$.

Our goal in this paper is twofold: (1) consider both primary and secondary usages as first-class citizens by proposing a privacy-preserving solution for computing multi-dimensional COUNTs over worker profiles and a task assignment algorithm based on recent affordable Private Information Retrieval (PIR) techniques, and (2) integrate well with other privacy-preserving algorithms possibly executed by a platform without jeopardizing the privacy guarantees by requiring our privacy model to be composable both with usual computational cryptographic guarantees provided by real-life encryption schemes and with classical differential privacy guarantees as well.

The two problems are not trivial. First, we focus on secondary usages. The problem of computing multi-dimensional COUNTs over distributed worker profiles in a privacy-preserving manner is not trivial. Interactive approaches - that issue a privacy-preserving COUNT query over the set of workers each time needed (e.g., a requester estimates the number of workers qualified for a given task) - are inadequate because the number of queries would be unbounded. This

\footnotetext{
${ }^{9}$ See, e.g., http://applymagicsauce.com/about-us

${ }^{10}$ For example, internal emails that were leaked from Deliveroo indicate that the geolocation system of Deliveroo was used internally for identifying the riders that participated to strikes against the platform.

https://www.lemonde.fr/culture/article/2019/09/24/

television-cash-investigation-a-la-rencontre-des-nouveaux-proletaires-du-web 6012758 3246.html

${ }^{11}$ In another example, an Uber executive claimed having tracked a journalist using the company geolocation system. https://tinyurl.com/y4cdvw45

12 https://eur-lex.europa.eu/eli/reg/2016/679/oj

13 https://www.caprivacy.org/

14 Note that limiting the information disclosed to the platform (i.e., perturbed information about worker profiles) relieves platforms from the costly task of handling personal data. The European GDPR indeed explicitely excludes anonymized data from its scope (see Article 4, Recital 26 https://gdpr-info.eu/recitals/no-26/).
} 
would lead to out-of-control information disclosure through the sequence of COUNTs computed [811]. Non-interactive approaches are a promising avenue because they compute, once for all and in a privacy-preserving manner, the static data structure(s) which are then exported and queried by the untrusted parties (e.g., platform, requesters) without any limit on the number of queries. More precisely, on the one hand, hierarchies of histograms are well-known data structures that support COUNT queries and that cope well with the stringent privacy guarantees of differential privacy [34]. However, they do not cope well with more than a few dimensions [34, whereas a worker profile may contain more skills (e.g., a dozen), and they require a trusted centralized platform. On the other hand, privacy-preserving spatial decompositions [941] are more tolerant to a higher number of dimensions but require as well a trusted centralized platform. Second, algorithms for assigning tasks to workers while providing sound privacy guarantees have been proposed as alternatives against naive spamming approaches - where all tasks are sent to all workers. However they are either based (1) on perturbation only (e.g., [5]) and suffer from a severe drop in quality or (2) on encryption only (e.g., [20]) but they do not reach realistic performances, or (3) they focus on the specific context of spatial crowdsourcing and geolocation data (e.g., [38]).

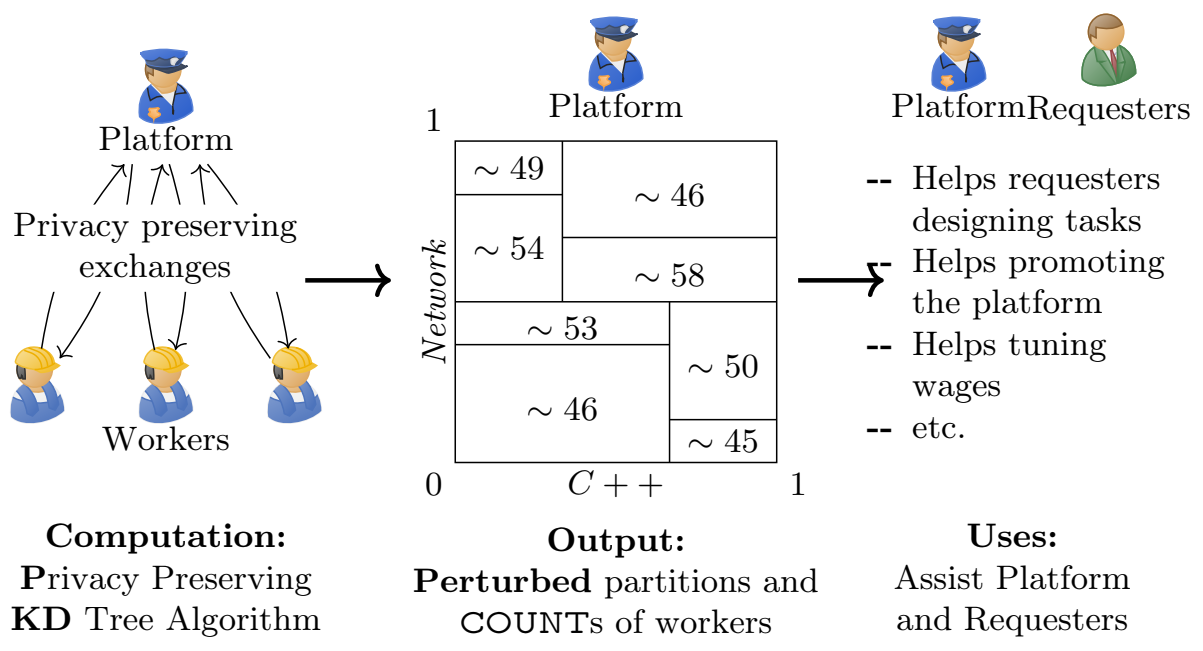

Fig. 1. Overview of the PKD algorithm: supporting secondary usages of worker profiles with privacy guarantees

Our Contribution. First, we propose to benefit from the best of the two noninteractive approaches described above by computing a privacy-preserving space partitioning of the worker profiles (for coping with their dimensionality) based on perturbed 1-dimensional histograms (for their nice tolerance to differentially 
private perturbations). We propose the Privacy-preserving KD-Tree algorithm (PKD for short, depicted in Figure 11, a privacy-preserving algorithm for computing a (perturbed) multi-dimensional distribution of skills of the actual population of workers. The PKD algorithm is distributed between mutually distrustful workers and an untrusted platform. It consists in splitting recursively the space of skills in two around the median (similarly to the $K D$-tree construction algorithm) based on the 1-dimensional histogram of the dimension being split, and it protects workers' profiles all along the computation by combining additively-homomorphic encryption together with differentially private perturbation. No raw worker profile is ever communicated, neither to the platform nor to other workers. The output of the PKD algorithm is a hierarchical partitioning of the space of skills together with the (perturbed) COUNT of workers per partition (see Figure 1). The PKD algorithm is complementary to privacy-preserving task assignment works and can be used in conjunction with them provided that the privacy models compose well. In particular, since our privacy model is a computational variant of differential privacy, the PKD algorithm composes well with state-of-the-art approaches [20[5] since they are based on usual computational cryptographic model or differential privacy model. Second, we propose to benefit from recent progresses in Private Information Retrieval techniques [2] in order to design a solution to task assignment that is both private and affordable. We perform an in-depth study of the problem of using PIR techniques for proposing tasks to workers, show that it is NP-Hard, and come up with the PKD PIR Packing heuristic that groups tasks together according to the partitioning output by the PKD algorithm. Obviously, the PKD PIR Packing heuristic composes well with the PKD algorithm.

More precisely, we make the following contributions:

1. We design the PKD algorithm, a distributed privacy-preserving algorithm for computing a multi-dimensional hierarchical partitioning of the space of skills within a population of workers.

2. We formally prove the security of the PKD algorithm against honest-butcurious attackers. The PKD algorithm is shown to satisfy a computational variant of differential privacy called the $\epsilon_{\kappa}$-SIM-CDP model. We provide a theoretical analysis of its complexity.

3. We provide an in-depth study of the problem of using PIR techniques for proposing tasks to workers and design the PKD PIR Packing heuristic that benefits from the partitioning computed by the PKD algorithm for grouping tasks together. We show that the PKD PIR Packing heuristic satisfies our privacy model.

4. We provide an extensive experimental evaluation of the PKD algorithm and of the PKD PIR Packing heuristic over synthetic and realistic data that demonstrates their quality and performance in various scenarios. Our realistic skills dataset is built from data dumps of StackExchange online forums.

The paper is organized as follows. Section 2 introduces the participant model, the security and privacy models, and the technical tools necessary in the rest of the paper. Section 3 describes the PKD algorithm in details and formally analyzes 
its cost and security. Section 4 studies the problem of using PIR techniques for task assignment, describes the PKD PIR Packing heuristic, and formally analyzes its security. We discuss some details on how to allow updates for both the PKD algorithm and the PKD PIR Packing heuristic in Section 6 Section 5 experimentally validates their quality and efficiency. In Section 7 , we survey the related work. Finally, Section 8 concludes and discusses interesting future works.

\section{Preliminaries}

\subsection{Participants Model}

Three types of participants collaborate together during our crowdsourcing process. Workers are interested in solving tasks that are relevant to their profiles; requesters propose tasks to be solved by relevant workers; and an intermediary, i.e., the platform.

A worker profile $p_{i} \in \mathcal{P}$ is represented by an $n$-dimensional vector of floats, where each float value $p_{i}[j] \in[0,1]$ represents the degree of competency of the worker $i$ with respect to the $j^{\text {th }}$ skill. The set of skills available and their indexes within workers' profiles is static and identical for all profiles.

A task $t_{k} \in \mathcal{T}$ is made of two parts. First, the metadata part is a precise description of the worker profiles that are needed for the task completion. More precisely it is an $n$-dimensional subspace of the space of skills. This work does not put any constraint on the kind of subspace described in the metadata part (e.g., hyper-rectangles, hyper-spheres, arbitrary set operators between subspaces). However, for the sake of concreteness, we focus below on metadata expressed as hyper-rectangles. More formally, the metadata $m_{k} \in \mathcal{M}$ of a task $t_{k} \in \mathcal{T}$ is an $n$-dimensional vector of ranges over skills where the logical connector between any pair of ranges is the conjunction. We call $m_{k}[j]$ the range of float values (between 0 and 1 ) for task $k$ and skill $j$. The second part of a task consists in the necessary information for performing the task and is represented as an arbitrary bitstring $\{0,1\}^{*}$. In this work, we essentially focus on the metadata part of tasks. We say that a worker and a task match if the point described by the worker profile belongs to the subspace described by the task metadata, i.e., worker $p_{i}$ and task $t_{k}$ match if and only if $\forall j \in[0, n-1]$, then $p_{i}[j] \in m_{k}[j]$.

We do not make strong assumptions on the resources offered by participants. Workers, requesters, and the platform are equipped with today's commodity hardware (i.e., the typical $\mathrm{CPU} /$ bandwidth/storage resources of a personal computer). However, we expect the platform to be available $24 / 7$ - contrary to workers or requesters - similarly to a traditional client/server setting.

We assume that all participants follow the honest-but-curious attack model in that they do not deviate from the protocol but make use of the information disclosed, in any computationally-feasible way, for inferring personal data. Workers may collude together up to a reasonable bound denoted by $\tau$ in the following. 


\subsection{Privacy Tools}

Computational Differential Privacy. Our proposal builds on two families of protection mechanisms: a differentially private perturbation scheme and a semantically secure encryption scheme. The resulting overall privacy model thus integrates the two families of guarantees together. The original $\epsilon$-differential privacy model [12] (Definition 11) applies to a randomized function $f$ and aims at hiding the impact of any possible individual value on the possible outputs of $f$. In our context, the function $f$ is the PKD algorithm. The $\epsilon$-differential privacy model requires that the probability that any worker profile $p_{i} \in \mathcal{P}$ participates to the computation of $\mathrm{f}$ be close to the probability that $p_{i}$ does not participate by an $e^{\epsilon}$ factor, whatever the output of $f$. $\epsilon$-differential privacy holds against information-theoretic adversaries (unlimited computational power).

Definition 1 ( $\epsilon$-differential privacy [12]). The randomized function $\mathrm{f}$ satisfies $\epsilon$-differential privacy, where $\epsilon>0$, if:

$$
\operatorname{Pr}\left[\mathrm{f}\left(\mathcal{P}_{1}\right)=\mathcal{O}\right] \leq e^{\epsilon} \cdot \operatorname{Pr}\left[\mathrm{f}\left(\mathcal{P}_{2}\right)=\mathcal{O}\right]
$$

for any set $\mathcal{O} \in$ Range(f) and any set of worker profiles $\mathcal{P}_{1}$ and $\mathcal{P}_{2}$ that differ in at most one profile.

The $\epsilon_{\kappa}$-SIM-CDP differential privacy relaxation [30] requires that the function actually computed be computationally indistinguishable from a pure (information theoretic) $\epsilon$-differentially private function to adversaries whose size is polynomial in the security parameter $\kappa \in \mathbb{N}$. The $\epsilon_{\kappa}$-SIM-CDP model is especially relevant in our context because we combine a differentially private perturbation scheme (information theoretic guarantees) together with an additively-homomorphic encryption scheme that provides computational security guarantees for performance reasons.

Definition 2 ( $\epsilon_{\kappa}$-SIM-CDP privacy [30] (simplified)). The randomized function $\mathbf{f}_{\kappa}$ provides $\epsilon_{\kappa}-S I M-C D P$ if there exists a function $\mathrm{F}_{\kappa}$ that satisfies $\epsilon$-differential privacy and a negligible function negl $(\cdot)$, such that for every set of worker profiles $\mathcal{P}$, every probabilistic polynomial time adversary $\mathrm{A}_{\kappa}$, every auxiliary background knowledge $\zeta_{\kappa} \in\{0,1\}^{*}$, it holds that:

$$
\left|\operatorname{Pr}\left[\mathrm{A}_{k}\left(\mathrm{f}_{\kappa}\left(\mathcal{P}, \zeta_{\kappa}\right)\right)=1\right]-\operatorname{Pr}\left[\mathrm{A}_{k}\left(\mathrm{~F}_{\kappa}\left(\mathcal{P}, \zeta_{\kappa}\right)\right)=1\right]\right| \leq \operatorname{negl}(\kappa)
$$

Achieving Differential Privacy with the Geometric Mechanism. A common mechanism for satisfying $\epsilon$-differential privacy with functions that output floats or integers consists in adding random noise to their outputs. In particular, the Geometric Mechanism (Definition 3) allows functions that output integers to be perturbed and to satisfy $\epsilon$-differential privacy, while maximizing utility for count queries as shown in [15].

Definition 3 (Geometric mechanism [15]). Let $\mathcal{G}$ denote a random variable following a two-sided geometric distribution, meaning that its probability density 
function is $g(z, \alpha)=\frac{1-\alpha}{1+\alpha} \alpha^{|z|}$ for $z \in \mathbb{Z}$. Given any function $\mathbf{f}: \mathbb{N}^{|\mathcal{X}|} \rightarrow \mathbb{Z}^{k}$ the Geometric Mechanism is defined as $M_{G}(x, f(),. \alpha)=\mathrm{f}(x)+\left(Y_{1}, \ldots, Y_{k}\right)$ where $Y_{i}$ are independent identically distributed random variables drawn from $\mathcal{G}\left(e^{-\epsilon / \Delta \mathrm{f}}\right)$, and $\Delta \mathrm{f}$ is its global sensivity $\Delta \mathrm{f}=\max _{\mathcal{P}_{1}, \mathcal{P}_{2}}\left\|\mathrm{f}\left(\mathcal{P}_{1}\right)-\mathrm{f}\left(\mathcal{P}_{2}\right)\right\|_{1}$ for all $\left(\mathcal{P}_{1}, \mathcal{P}_{2}\right)$ pairs of sets of worker profiles s.t. $\mathcal{P}_{2}$ is $\mathcal{P}_{1}$ with one profile more.

Intuitively, a distribution is said to be infinitely divisible if it can be decomposed as a sum of an arbitrary number of independent identically distributed random variables. This property allows to distribute the generation of the noise over a set of participants. It is valuable in contexts such as ours where no single trusted party, in charge of generating the noise, exists. Definition 4 below formalizes the infinite divisibility property, and Theorem 1 shows that the two-sided geometric distribution is infinitely divisible.

Definition 4 (Infinite Divisibility). A probability distribution with characteristic function $\psi$ is infinitely divisible if, for any integer $n \geq 1$, we have $\psi=\phi_{n}^{n}$ where $\phi_{n}$ is another characteristic function. In other words, a random variable $Y$ with characteristic function $\psi$ has the representation $Y \stackrel{d}{=} \sum_{i=1}^{n} X_{i}$ for some independent identically distributed random variables $X_{i}$.

Theorem 1 (Two-sided Geometric Distribution is Infinitely Divisible). Let $Y$ follow two-sided geometric distribution with probability density function $d(z, \epsilon)=\frac{1-\epsilon}{1+\epsilon} \epsilon^{|z|}$ for any integer $z$. Then the distribution of $Y$ is infinitely divisible. Furthermore, for every integer $n \geq 1$, representation of definition 4 holds. Each $X_{i}$ is distributed as $X_{1 n}-X_{2 n}$ where $X_{1 n}$ and $X_{2 n}$ are independent identically distributed random variable with negative binomial distribution, with probability density function $g(k, n)=\left(\begin{array}{c}k-1+1 / n \\ k\end{array}\right)(1-\alpha)^{k} * \alpha^{1 / n}$.

To prove this result, we will use a similar result for the geometric distribution.

Theorem 2 (Geometric Distribution is Infinitely Divisible [36]). Let $Y$ have a geometric distribution with probability density function $f(k, \alpha)=(1-\alpha) *$ $\alpha^{k}$ for $k \in \mathbb{N}$. Then the distribution of $Y$ is infinitely divisible. Furthermore, for every integer $n \geq 1$, representation of 4 holds. Each $X_{i}$ is distributed as $X_{n}$ where $X_{n}$ are independent identically distributed random variable with negative binomial distribution, with probability density function $g(k, n, \alpha)=\left(\begin{array}{c}k-1+1 / n \\ k\end{array}\right)(1-\alpha)^{k} \alpha^{1 / n}$.

Proof. Using this theorem, proving that a two-sided geometric distribution with density function $d(z, \alpha)=\frac{1-\alpha}{1+\alpha} \alpha^{|z|}$ is equal to the difference between two independent identically distributed geometric distributions with density function $f(k, \alpha)=(1-\alpha) \alpha^{k}$ is enough to deduce the result. Let $X_{+}$and $X_{-}$be two such random variables.

$$
P\left(X_{+}-X_{-}=z\right)=\left\{\begin{array}{l}
\text { if } z \geq 0 \\
\sum_{j=0}^{\infty}\left((1-\alpha) \alpha^{z+j}\right)\left((1-\alpha) \alpha^{j}\right) \\
\text { if } z<0 \\
\sum_{j=0}^{\infty}\left((1-\alpha) \alpha^{j}\right)\left((1-\alpha) \alpha^{-z+j}\right)
\end{array}\right.
$$




$$
\begin{aligned}
P\left(X_{+}-X_{-}=z\right) & =\sum_{j=0}^{\infty}(1-\alpha)^{2} \alpha^{|z|+2 j} \\
& =(1-\alpha)^{2} \alpha^{|z|} \sum_{j=0}^{\infty}\left(\alpha^{2}\right)^{j} \\
& =(1-\alpha)^{2} \alpha^{|z|} \frac{1}{1-\alpha^{2}} \\
& =\frac{(1-\alpha)^{2}}{(1-\alpha)(1+\alpha)} \alpha^{|z|} \\
& =\frac{1-\alpha}{1+\alpha} \alpha^{|z|} \\
& =P(Y=z)
\end{aligned}
$$

for $Y$ a random variable with a two-sided geometric distribution with parameter $\alpha$.

Finally, Theorem 3 states that differential privacy composes with itself gracefuly.

Theorem 3 (Sequential and Parallel Composability [13]). Let $\mathbf{f}_{i}$ be a set of functions such that each provides $\epsilon_{i}$-differential privacy. First, the sequential composability property of differential privacy states that computing all functions on the same dataset results in satisfying $\left(\sum_{i} \epsilon_{i}\right)$-differential privacy. Second, the parallel composability property states that computing each function on disjoint subsets provides $\max \left(\epsilon_{i}\right)$-differential privacy.

Additively-Homomorphic Encryption. Additively-homomorphic encryption schemes essentially allow to perform addition operations over encrypted data. Any additively-homomorphic encryption scheme fits our approach provided that it satisfies the following properties. First, it must provide semantic security guarantees. Stated informally, this property requires that given a ciphertext, the public encryption key, and possible auxiliary information about the plaintext, then no polynomial-time algorithm is able to gain non-negligible knowledge on the plaintext [16. Second, it must be additively-homomorphic. Informally, given $a$ and $b$ two integers, $E$ the encryption function, $X$ the encryption key, $K$ the decryption key, D the decryption function, and $+_{h}$ the homomorphic addition operator, then $\mathrm{D}_{K}\left(\mathrm{E}_{X}(a)+{ }_{h} \mathrm{E}_{X}(b)\right)==a+b$. Third, the scheme must support non-interactive threshold decryption. We additionally use this optional property, available in some schemes (e.g., 3110]). It allows the decryption key to be split in $n_{K}$ key-shares $K_{i}$ such that a complete decryption requires to perform independently $T \leq n_{K}$ partial decryptions by distinct key-shares. Note that in a typical key generation setting, pairs of keys are generated once and for all by a non-colluding, independent entity.

Paillier cryptosystem [31] and its Damgard-Jurik generalization [10] are instances of encryption schemes that provide the desired properties and are widely available. We refer the interested reader to the original papers for details.

Private Information Retrieval. In a nutshell, Private Information Retrieval (PIR) techniques allow a client to download binary objects (e.g., a record, a movie) stored on a server in a library of objects, without revealing to the server which of the binary objects has been downloaded. We call this function the PIR-get 
function. Emerging PIR protocols are now affordable and able to cope with the latency constraints of real-life scenarios (e.g., in media consumption scenarios [17/2/7]). Our approach makes use of the security guarantees of PIR techniques. In this paper, for concreteness, we consider a PIR protocol based on additivelyhomomorphic encryption called XPIR [2]. It is part of the computational PIR family of protocols, that provides computational security guarantees. However our approach could use other protocols, that provide different tradeoffs efficiency/security (e.g., an information-theoretic PIR protocol such as [7 that uses efficient bitwise XOR operators, provides information-theoretic security, but assumes no collusion between several supporting servers).

XPIR [2] considers a library $\mathcal{L}$ of $n$ binary objects, called items in the following. All items are assumed to share the same length in bits, denoted $l$. The library is stored as a matrix of $y$-bit integers: $\mathcal{L} \in\left(\{0,1\}^{y}\right)^{n \times(l / y)}$. XPIR uses an additivelyhomomorphic cryptosystem (see above) and implements the PIR-get function as follows. XPIR assumes that each item has a unique id, and that clients know the list of ids of the existing items in $\mathcal{L}$. Now a client wants to retrieve the item of id $i$ and thus calls PIR-get(i). First, it instanciates a vector of $n$ bits, initializes all bits to $0 \mathrm{~s}$, sets to 1 the bit at id $i$, encrypts each bit separately, and sends the resulting encrypted vector - denoted $c$ - to the server. Second, for all $j$ in $[1, l / y]$, the server computes $r_{j}=\prod_{i=1}^{n} c[i]^{L_{i, j}}$ (recall that the product between two encrypted integers is the way the additively-homomorphic addition operator $+_{h}$ is performed by the underlying cryptosystem) and sends it back to the client. Each $r_{j}$ is thus actually a sum of (1) encrypted 0s (corresponding to the encrypted $0 \mathrm{~s}$ in $c$ ) and (2) an encrypted bit-subsequence of the requested binary object (corresponding to the encrypted 1 in $c$ ). Third and finally, the client decrypts each $r_{j}$ received - obtaining hence the various bit-subsequences of the item requested - and concatenates them to obtain the complete bitstring.

It is to be noticed that three main parameters may affect PIR efficiency: the size of the library itself, that has to be read for each call to the PIR-get function, the size of items, that impacts the size of downloads (multiplied by an expansion factor, the ratio between the size of an encrypted value and the clear value), and the number of items (again, multiplied by the expansion factor), for the size of upload of the request.

\subsection{Space Partitionning based on KD-Trees}

A KD-Tree [4 is a well-known data structure designed for partitioning datasets in k-dimensional balanced partitions. It is constructed by recursively dividing the space in two around the median. It is widely used to index data. Moreover, it contains valuable information about the data distribution (it is balanced) without sizing individual data points.

\subsection{Quality Measure}

We evaluate the quality of the (perturbed) output of the PKD algorithm by measuring the loss of accuracy resulting from its use rather than using non- 
protected raw profiles. As stated in Definition 5 given a set of tasks $T$, we compute the average absolute error between (1) the approximate number of workers matching with each task $t \in \mathcal{T}$ according to the (perturbed) partitions and counts and (2) the exact non-protected number of matching workers. Note that the error comes from the perturbation used for satisfying differential privacy but also from the inherent approximation due to the use of a coarse grain data structure (partitions and counts) synthesizing raw data.

Definition 5 (Quality). Given a set of worker profiles $\mathcal{P}$, a set of tasks $\mathcal{T}$, and, for each task $t \in \mathcal{T}$, the real number of workers matching with it $t_{\text {match }}$ and its approximated value $\widetilde{t_{\text {match }}}$ according to the perturbed distribution we provide. We compute the quality of the distribution as

$$
Q=\frac{1}{|\mathcal{T}|} \sum_{t \in \mathcal{T}} \frac{\left|t_{\text {match }}-\widehat{t_{\text {match }}}\right|}{t_{\text {match }}}
$$

We also measure the quality of our assignment by using precision, to size the number of tasks that are uselessly downloaded by workers, as seen in Definition 6 . Note that in our context, all matching tasks will be downloaded, such that a recall measure is not relevant as it will always be equal to 1 .

Definition 6 (Precision). For a given assignment, we call precision the fraction of downloaded tasks that match with the worker. This precision is computed as:

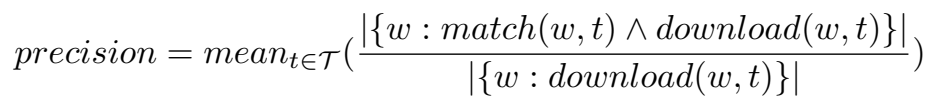

\section{The PKD Algorithm}

Our proposal comes from a rethinking of the centralized version of the $K D$ Tree construction algorithm [4, which is essentially a recursive computation of medians. In our setting, each worker holds its own, possibly sensitive, profile and no single centralized party is trusted. Centralizing the profiles of workers in order to compute a KD-Tree over them is therefore not possible. A naive approach could make use of an order-preserving encryption scheme [1] (OPE), but these schemes are well-known for their low security level [6] and especially their inherent weaknesses against frequency analysis attacks. We rather favor sound privacy guarantees - without sacrificing efficiency - by approaching the median through the computation of histograms, with a computation distributed between workers and the platform. Similarly to its centralized counterpart, each iteration of our recursive algorithm divides the space of skills in two around the median (of one dimension at a time) given a perturbed histogram representing the distribution of a single skill in the crowd. For simplicity, the current version of the algorithm terminates after a fixed number of splits, but more elaborate termination criteria can be defined. 


\subsection{Computing Private Medians}

From Private Sum to Private Histogram. We start by explaining how to perform differentially private sums based on noise-shares and additively-homomorphic additions. It allows the platform to get the result of the addition of a single bin over $n$ different workers while satisfying differential privacy. We then show that this function is a sufficient building block for computing perturbed histograms.

Let us consider a fixed $\epsilon>0$, a maximum size of collusion $\tau \in \mathbb{N}, \tau>0$, a set of workers $\mathcal{P}$ (of size $|\mathcal{P}|$ ), and a single bin $b_{i}$ associated to a range $\phi$. Each worker $p_{i}$ holds a single private local value, i.e., her skill on the dimension that is currently being split. Initially, the bin $b_{i}$ is set to 0 on all workers. Only the workers whose local values fall within the range of the bin $b_{i}$ set $b_{i}$ to 1 . Our first goal is to compute the sum of the bins $b_{i}$ of all workers $p_{i} \in \mathcal{P}$ such that no set of participant smaller than $\tau$ can learn any information that has not been perturbed to satisfy $\epsilon$-differential privacy.

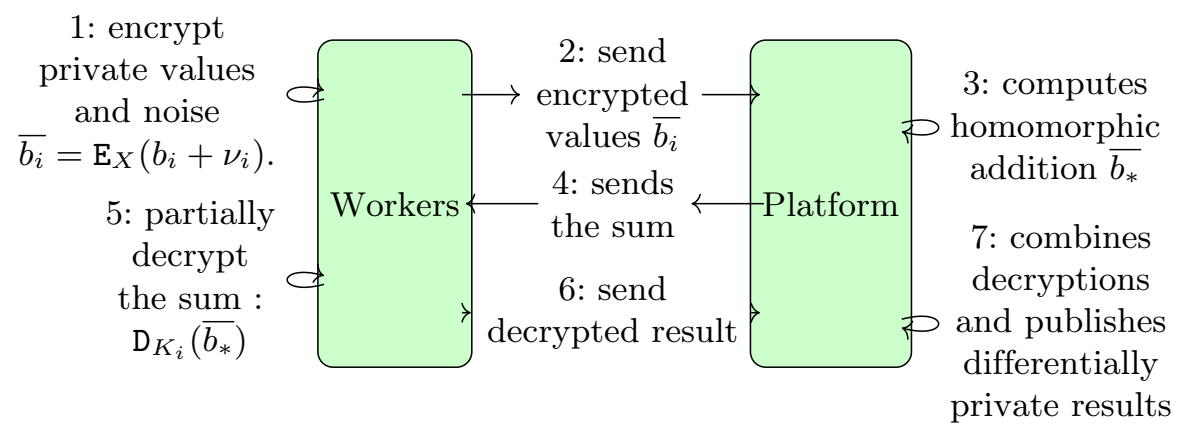

Fig. 2. Computing a Private Sum

The privacy-preserving sum algorithm, depicted in Fig. 2. considers that keys have been generated and distributed to workers, such that $T>\tau$ key-shares are required for decryption (see Section 2.2). The algorithm consists in the following steps:

Step 1 (each worker) - Perturbation and Encryption First, each worker perturbs its value by adding a noise-share, denoted $\nu_{i}$, to it. Noise-shares are randomly generated locally such that the sum of $|\mathcal{P}|-\tau$ shares satisfies the two-sided geometric distribution (see Definition 3 for the geometric distribution, and Theorem 1 for its infinite divisibility). Note that noiseshares are overestimated ${ }^{15}$ to guarantee that the final result is differentially private even for a group of up to $\tau$ workers sharing their partial knowledge

$\overline{15}$ We require that the sum of $|\mathcal{P}|-\tau$ noise-shares be enough to satisfy differential privacy but we effectively sum $|\mathcal{P}|$ noise-shares. Note that summing more noise-shares than necessary does not jeopardize privacy guarantees. 
of the total noise (their local noise-share). Each worker then encrypts $b_{i}+\nu_{i}$ by the additively-homomorphic encryption scheme in order to obtain $\overline{b_{i}}$ : $\overline{b_{i}}=\mathrm{E}_{X}\left(b_{i}+\nu_{i}\right)$.

Step 2 (platform) - Encrypted Sum The platform sums up together the encrypted values received : $\overline{b_{*}}=\sum_{\forall i} \overline{b_{i}}$ where the sum is based on the additively-homomorphic addition $+_{h}$.

Step 3 (subset of workers) - Decryption The platform finally sends the encrypted sum $\overline{b_{*}}$ to at least $T$ distinct workers. Upon reception, each worker partially decrypts it based on her own key-share - $\mathrm{D}_{K_{i}}\left(\overline{b_{*}}\right)$ - and sends it back to the platform. The platform combines the partial decryptions together and obtains $\widetilde{b_{*}}$, i.e., the differentially private sum of all private bins $b_{i}$.

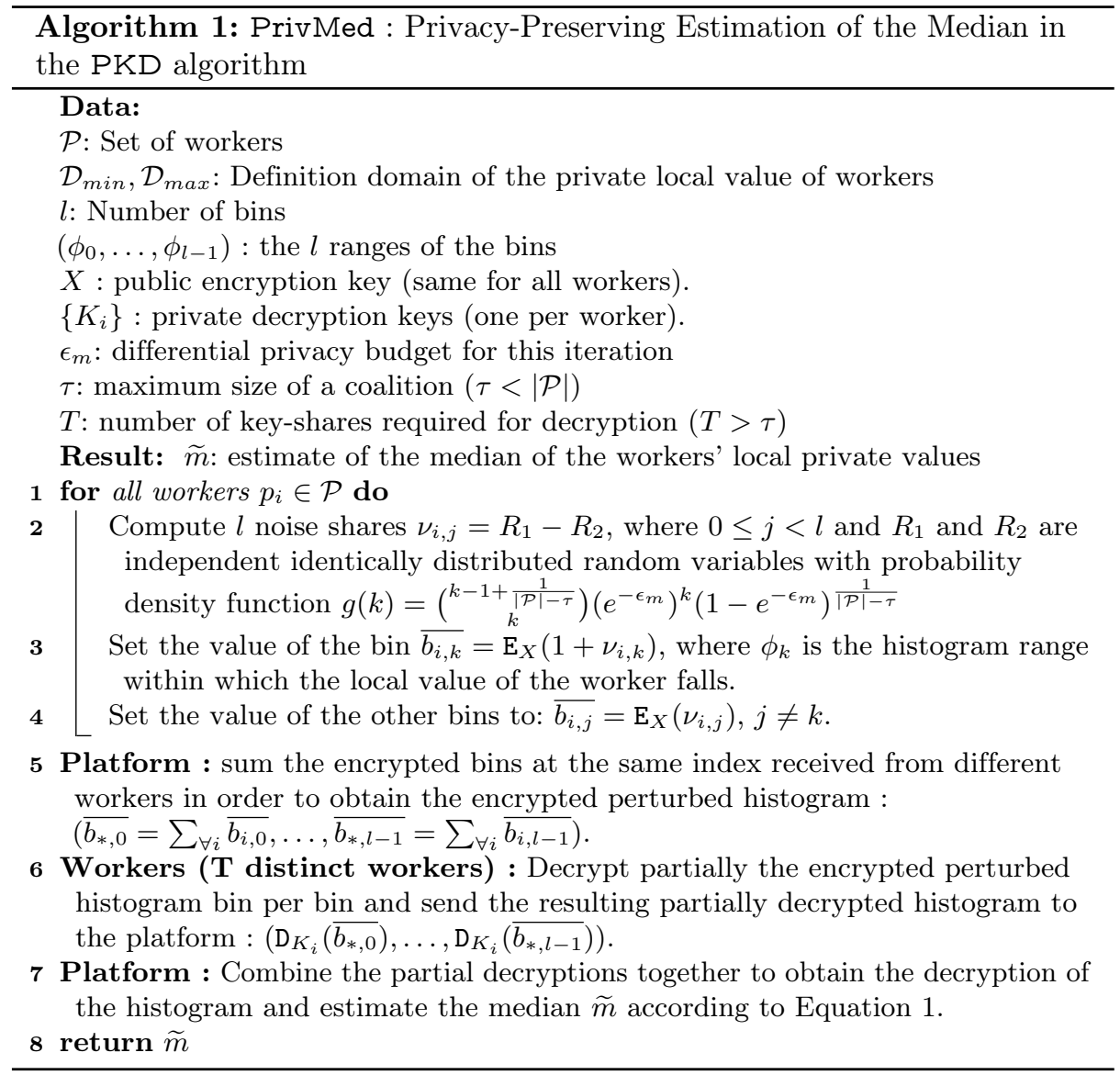

Now, assuming that the histogram format is fixed beforehand - i.e., number $l$ of bins and ranges $\left(\phi_{0}, \ldots, \phi_{l-1}\right)$ - it is straightforward to apply the private sum 
algorithm on each bin for obtaining the perturbed histogram based on which the median can then be computed. For example, in order to get a histogram representing the distribution of skill values for, e.g., Python programming, and assuming a basic histogram format - e.g., skill values normalized in $[0,1], l=10$ bins, ranges $\left(\phi_{0}=\left[0,0.1\left[, \ldots, \phi_{9}=[0.9,1]\right)\right.\right.$ - it is sufficient to launch ten private sums to obtain the resulting perturbed 10-bins histogram.

PrivMed: privacy-preserving median computation. The histogram computed based on the privacy-preserving sum algorithm can be used by the platform to estimate the value of the median around which the split will be performed. When by chance the median falls precisely between two bins (i.e., the sum of the bins on the left is exactly $50 \%$ of the total sum, same for the bins on the right) its value is exact. But when the median falls within the range of one bin (i.e., in any other case), an additional hypothesis on the underlying data distribution within the bin must be done in order to be able to estimate the median. For simplicity, we will assume below that the distribution inside each bin is uniform but a more appropriate distribution can be used if known.

$$
\widetilde{m}=\mathcal{D}_{\min }+\frac{\mathcal{D}_{\max }-\mathcal{D}_{\text {min }}}{l} \cdot\left(k+\frac{1}{2}+\frac{\theta_{>}-\theta_{<}}{2 \cdot \widetilde{b_{*, k}}}\right)
$$

The resulting PrivMed algorithm is detailed in Algorithm 1

Let's consider the histogram obtained by the private sum algorithm. It is made of $l$ bins denoted $\left(\widetilde{b_{*, 0}}, \ldots, \widetilde{b_{*, l-1}}\right)$, and each bin $\widetilde{b_{*, j}}$ is associated to a range $\phi_{j}$. The ranges partition a totally ordered domain ranging from $\mathcal{D}_{\min }$ to $\mathcal{D}_{\max }$ (e.g., from $\mathcal{D}_{\text {min }}=0$ to $\mathcal{D}_{\text {max }}=1$ on a normalized dimension that has not been split yet). Let $\phi_{k}$ denote the range containing the median, $\theta$ denote the sum of all the bins - i.e., $\theta=\sum_{i<l} \widetilde{b_{*, i}}-$ and $\theta_{<}\left(\right.$resp. $\left.\theta_{>}\right)$the sum of the bins that are strictly before (resp. after) $\widetilde{b_{*, k}}-$ i.e., $\theta_{<}=\sum_{i<k} \widetilde{b_{*, i}}\left(\right.$ resp. $\theta_{>}=\sum_{i>k} \widetilde{b_{*, i}}$ ). Then, an estimation $\widetilde{m}$ of the median can be computed as follow:

\subsection{Global Execution Sequence}

Finally, the Privacy-preserving KD-Tree algorithm, PKD for short, performs the median estimation described above iteratively until it stops and outputs (1) a partitioning of the space of skills together with (2) the perturbed number of workers within each partition. The perturbed number of workers is computed by using an additional instantiation of the private sum algorithm when computing the private medians ${ }^{17}$. We focus below on the setting up of the parameters of the various iterations, and on the possible use of the resulting partitions and counts by the requesters. An overview is given in Algorithm 2

${ }^{16}$ Note that in the specific case where the median falls within a bin equal to 0 (i.e., $\widetilde{b_{*, k}}=0$ ), then any value within $\phi_{k}$ is equivalent.

17 Note that the perturbed histograms could have been used for computing these counts but using a dedicated count has been shown to result in an increased precision. 
Main Input Parameters. Intuitively, the privacy budget $\epsilon$ input of the PKD algorithm sets an upper bound on the information disclosed along the complete execution of the algorithm - it must be shared among the various data structures disclosed. Thus, each iteration inputs a portion of the initial privacy budget such that the sum of all portions remains lower than or equal to $\epsilon$ - see the composability property in Theorem 3 . Computing a good budget allocation in a tree of histograms is a well-known problem tackled by several related works 9133. In this work, we simply rely on existing privacy budget distribution methods. For example, based on [9], $\epsilon$ is divided as follows. First, $\epsilon$ is divided in two parts: one part, denoted $\epsilon^{m}$, is dedicated to the perturbations of the medians computations (i.e., the bins of the histograms), and the other part, denoted $\epsilon^{c}$, is dedicated to the perturbation of the number of workers inside each partition. Second, a portion of each of these parts is allocated to each iteration $i$ as follows. For each iteration $i$ such that $0 \leq i \leq h$, where $h$ is the total number of iterations (i.e., the height of the tree in the KD-Tree analogy), the first iteration is $h$ (i.e., the root of the tree) and the last one is 0 (i.e., the leaves of the tree) :

$$
\begin{gathered}
\epsilon_{i}^{c}=2^{(h-i) / 3} \epsilon^{c} \frac{\sqrt[3]{2}-1}{2^{(h+1) / 3}-1} \\
\epsilon_{i}^{m}=\frac{\epsilon^{m}}{h}
\end{gathered}
$$

Note that similarly to [9], we set the distribution of $\epsilon$ between $\epsilon^{c}$ and $\epsilon^{m}$ as follows: $\epsilon^{c}=0.7 \cdot \epsilon$ and $\epsilon^{m}=0.3 \cdot \epsilon$. Other distributions could be used.

The PKD algorithm stops after a fixed number of iterations known beforehand. Note that more elaborate termination criteria can be defined (e.g., a threshold on the volume of the subspace or on the count of worker profiles contained). The termination criteria must be chosen carefully because they limit the number of splits of the space of skills and consequently the number of dimensions of worker profiles that appear in the final subspaces. Ideally, the termination criteria should allow at least one split of all dimensions. However, this may not be possible or suitable in practice because of the limited privacy budget. In this case, similarly to a composite index, a sequence of priority dimensions must be chosen so that the algorithm splits them following the order of the sequence. The dimensions that are not part of the sequence will simply be ignored. Note that the number of dimensions in worker profiles, and their respective priorities, is closely related to the application domain (e.g., How specific does the crowdsourcing process need to be ?). In this paper, we make no assumption on the relative importance of dimensions.

Post-Processing the Output. Considering the successive splits of partitions, we can enhance the quality of the counts of workers by exploiting the natural constraints among the resulting tree of partitions : we know that the number of workers in a parent partition must be equal to the number of workers in the union of its children. Constrained inference techniques have already been studied as a post-processing step to improve the quality of trees of perturbed histograms, 


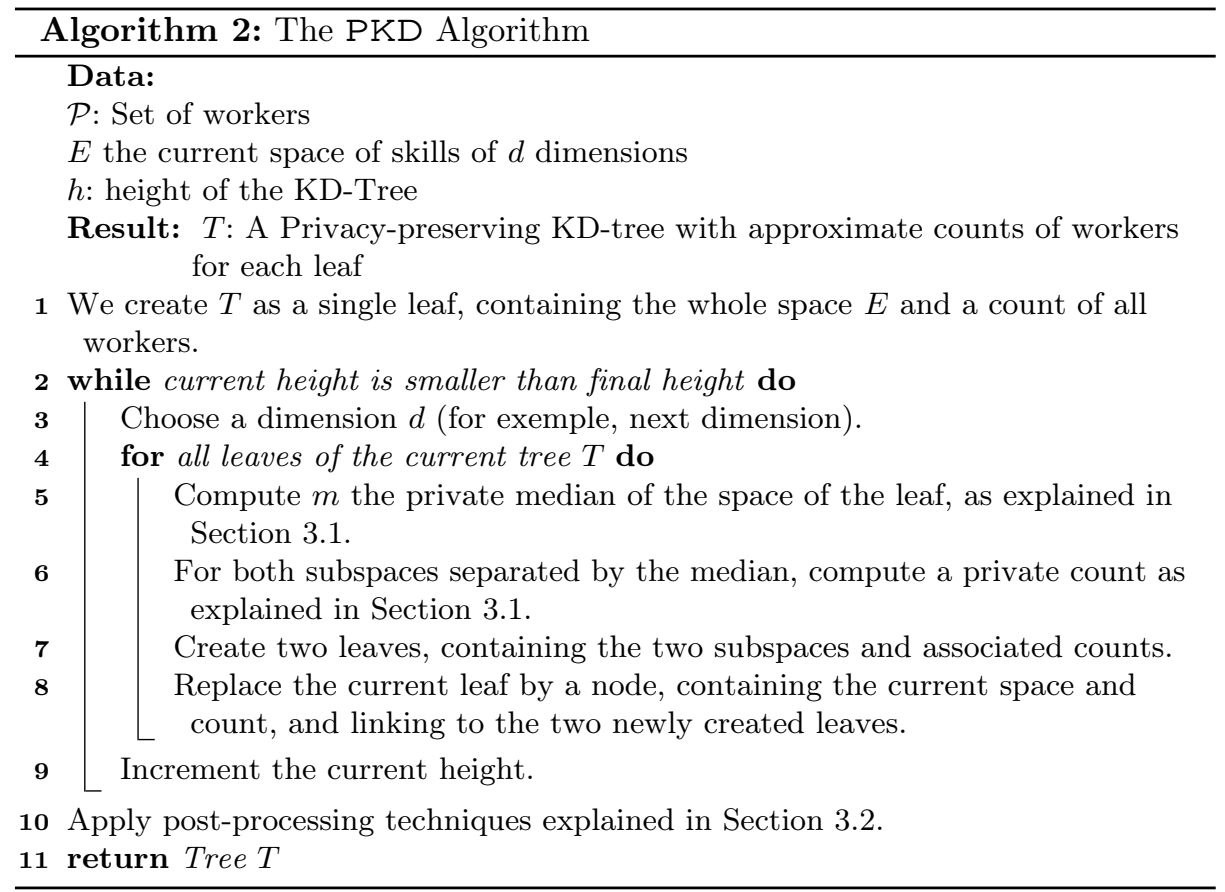

first in [19] and then improved in [9] which adapts the method to non-uniform distribution of budget. These constrained inference techniques can be used in our context in a straightforward manner in order to improve on the quality of the resulting partitioning. We refer the interested reader to [19]9] for details.

\subsection{Complexity Analysis}

We evaluate here the complexity of the PKD algorithm with respect to the number of encrypted messages computed and sent both to and by the platform. The results are summed up in Table 1 .

The first step to consider is the number of partitions created in the KD-tree. Seen as an index (with one leaf for each point), the construction of a KD-tree requires $2^{h+1}-1$ nodes, including $2^{h}-1$ internal nodes, where $h$ is the maximum height of the KD-Tree. For each node, an encrypted sum is performed, and for each internal node, a histogram is additionally computed, which require $l$ sums, for a total of $\left(2^{h+1}-1\right)+\left(l \cdot\left(2^{h}-1\right)\right)$ sums. These counts all require the participation of every worker: for each count, $|\mathcal{P}|$ encrypted messages are computed and sent.

The platform also sends back encrypted messages for each sum, for decryptions to be performed. For each sum, it sends at least $T$ times the homomorphically computed sum, where $T$ is the threshold number of key-shares required for decryption. For simplicity, we assume that the platform sends the cyphertexts to 
$T$ workers (these are the only encrypted messages that have to be sent to workers during this protocol). Each contacted worker then answers by an encrypted value (the partial decryption). As a conclusion, the total number of encrypted values sent by the workers to the platform $\mathcal{M}_{\Sigma w}$ is:

$$
\mathcal{M}_{\Sigma w}=(|\mathcal{P}|+T) \cdot\left(l \cdot\left(2^{h}-1\right)+\left(2^{h+1}-1\right)\right)
$$

However, as our computation is distributed among all workers, each worker only sends fewer encrypted messages on average $\overline{\mathcal{M}_{w}}$.

$$
\overline{\mathcal{M}_{w}}=\left(1+\frac{T}{|\mathcal{P}|}\right) \cdot\left(l \cdot\left(2^{h}-1\right)+\left(2^{h+1}-1\right)\right)
$$

Finally, the platform sends $\mathcal{M}_{p f}$ encrypted messages.

$$
\mathcal{M}_{p f}=T \cdot\left(l \cdot\left(2^{h}-1\right)+\left(2^{h+1}-1\right)\right)
$$

\begin{tabular}{|l|l|}
\hline To the platform & $(|\mathcal{P}|+T) \cdot\left(l \cdot\left(2^{h}-1\right)+\left(2^{h+1}-1\right)\right)$ \\
\hline By worker $(\operatorname{avg})$ & $\left(1+\frac{T}{|\mathcal{P}|}\right) \cdot\left(l \cdot\left(2^{h}-1\right)+\left(2^{h+1}-1\right)\right)$ \\
\hline By the platform & $T \cdot\left(l \cdot\left(2^{h}-1\right)+\left(2^{h+1}-1\right)\right)$ \\
\hline
\end{tabular}

Table 1. Number of encrypted messages sent. $\mathcal{P}$ is the set of workers, $T$ the number of partial keys required for decryption, $h$ the depth of the KD-tree, and $l$ the number of bins per median

\subsection{Security Analysis}

The only part of the PKD algorithm that depends on raw data is the private sum. The security analysis thus focuses on proving that a single private sum is secure, and then uses the composability properties (see Theorem 3). Theorem 4 proves that the privacy-preserving sum algorithm is secure. We use this intermediate result in Theorem 5 to prove the security of the complete PKD algorithm.

Theorem 4 (Security of the privacy-preserving sum algorithm). The privacy-preserving sum algorithm satisfies $\epsilon_{\kappa}-S I M-C D P$ privacy against coalitions of up to $\tau$ participants.

Proof. (sketch) First, any skill in a profile of a participating worker is first summed up locally with a noise-share, and then encrypted before being sent to the platform. We require the encryption scheme to satisfy semantic security, which means that no computationally-bounded adversary can gain significant knowledge about the data that is encrypted. In other words, the leak due to communicating an encrypted data is negligible. Second, the homomorphically-encrypted additions performed by the platform do not disclose any additional information. Third, 
the result of the encrypted addition is decrypted by combining $T>\tau$ partial decryptions, where each partial decryption is performed by a distinct worker. The threshold decryption property of the encryption scheme guarantees that no coalition of participants smaller than $\tau$ can decrypt an encrypted value, and the honest-but-curious behaviour of participants guarantees that no other result but the final one will be decrypted (e.g. the platform does not ask for a decryption of a value that would not have been sufficiently perturbed). The final sum consists in the sum of all private values, to which are added $|\mathcal{P}|$ noise-shares. These shares are computed such that the addition of $|\mathcal{P}|-\tau$ shares is enough to satisfy $\epsilon$-differential privacy. Thanks to the post-processing property of differential privacy, adding noise to a value generated by a differentially-private function does not impact the privacy level. The addition of $\tau$ additional noise-shares consequently allow to resist against coalitions of at most $\tau$ participants without thwarting privacy. As a result, since the privacy-preserving sum algorithm is the composition of a semantically secure encryption scheme with an $\epsilon$-differentially private function, it is computationally indistinguishable from a pure differentially private function, and consequently satisfies $\epsilon_{\kappa}$-SIM-CDP privacy against coalitions of up to $\tau$ participants.

Theorem 5 (Security of the PKD algorithm). The PKD algorithm satisfies $\epsilon_{\kappa}-S I M-C D P$ privacy against coalitions of up to $\tau$ participants.

Proof. (sketch) In the PKD algorithm, any collected information is collected through the PrivMed algorithm based on the privacy-preserving sum algorithm. Since (1) the privacy-preserving sum algorithm satisfies $\epsilon_{\kappa}$-SIM-CDP (see Theorem (4) against coalitions of up to $\tau$ participants, (2) $\epsilon_{\kappa}$-SIM-CDP is composable (see Theorem 3), and (3) the privacy budget distribution is such that the total consumption does not exceed $\epsilon$ (see Section 3.2), it follows directly that the PKD algorithm satisfies $\epsilon_{\kappa}$-SIM-CDP against coalitions of up to $\tau$ participants.

\section{Privacy-Preserving Task Assignement}

Once the design of a task is over, it must be assigned to relevant workers and delivered. Performing that while satisfying differential privacy and at the same time minimizing the number of downloads of the task's content is surprisingly challenging. We already discarded in Section 1, for efficiency reasons, the spamming approach in which each task is delivered to all workers. More elaborate approaches could try to let the platform filter out irrelevant workers based on the partitioned space output by the PKD algorithm (see the Section 3). The partitioned space would be used as an index over workers in addition to its primary task design usage. For example, workers could subscribe to their areas of interest (e.g., by sending an email address to the platform together with the area of interest) and each task would be delivered to a small subset of workers only according to its metadata and to the workers' subscriptions. However, despite their appealing simplicity, these platform-filtering approaches disclose unperturbed information about the number of workers per area, which breaks differential 
privacy, and fixing the leak seems hard (e.g., random additions/deletions of subscriptions, by distributed workers, such that differential privacy is satisfied and the overhead remains low).

We propose an alternative approach, based on Private Information Retrieval (PIR) techniques, to diminish the cost of download on the workers side, while preserving our privacy guarantees.

\subsection{PIR for Crowdsourcing: challenges and naive approaches}

The main challenge in applying PIR in our context consists in designing a PIRlibrary such that no information is disclosed during the retrieval of information, and performance is affordable in real-life scenarios. To help apprehending these two issues, we here present two naive methods that break these conditions and show two extreme uses of PIR: one efficient but unsecure, the other is secure but unefficient.

A first PIR-based approach could consist in performing straightforwardly a PIR protocol between the workers and the platform, while considering the PIR-library as the set of tasks itself. The platform maintains a key-value map that stores the complete set of tasks (the values, bitstrings required to perform the tasks) together with a unique identifier per task (the keys), together with their metadata. The workers download the complete list of tasks identifiers and metadata, select locally the identifiers associated to the metadata that match with their profiles, and launch one PIR-get function on each of the selected identifiers. However, this naive approach leads to blatant security issues through the number of calls to the PIR-get function. Indeed, in some cases, the platform could deduce the precise number of workers within a specific subspace of the space of skills: with the knowledge of the number of downloads for each worker ${ }^{18}$ it is possible to deduce, for each $k$, the number of workers downloading $k$ tasks. From that, the platform can deduce that the number of workers located in subspaces where $k$ tasks intersect together, and therefore precise information on their skills. This information, kept undisclosed thanks to the PKD algorithm, breaks differential privacy guarantees.

A secure but still naive approach could be to consider the power set of the set of tasks as the PIR-library, with padding to all file such that they are all the same size (in bits). After this, a worker chooses the PIR-object corresponding to the set of tasks she intersects with, and uses PIR-get on it. Although this method prevents the previously observed breach to appear (all behaviours are identical to the platform since everyone downloads exactly one PIR-object, and all PIR-objects are of the same size), this method would lead to extremely poor results: as every object of the library is padded to the biggest one, and the biggest set of the super set of tasks is the set of tasks itself, this algorithm is even worse

\footnotetext{
${ }_{18}$ Even if the identity of workers is not directly revealed, it is possible to match downloads together to break unlinkability and deduce these downloads come from the same individual, for example by using the time of downloads, cookies or other identification techniques
} 
than the spamming approach (everyone downloads at least as much as the sum of all tasks, with computation overheads).

These two naive uses of PIR illustrate two extreme cases: the first one shows that using PIR is not sufficient to ensure privacy, and the second one illustrates that a naive secure use can lead to higher computation costs than the spamming approach. In the following, we introduce a method to regroup tasks together, such that each worker downloads the same number of PIR items (to achieve security), while mitigating performance issues by making these groups of tasks as small as possible.

\subsection{PIR partitioned packing}

The security issue showed in the naive PIR use comes from the fact that the number of downloads directly depends on the profiles of workers. Indeed, as the platform has access to the number of downloads, this link leaks information about workers' skills. In order to break this link, we propose to ensure that each worker downloads the same number of items, whatever their profile is. For simplicity, we fix this number to $1^{19}$ and call packing a PIR library that allows each worker to retrieve all their tasks with only one item, and bucket an item of such a library, as seen in Definition 7. We prove in Theorem 6 that any packing fulfills our security model.

We can now formalize the conditions that a PIR library must fulfill in order to both satisfy privacy and allow any worker to download all the tasks she matches with.

\section{Definition 7 (Packing, Bucket).}

A packing $L$ is a PIR library which fulfills the following conditions:

1. Security condition Each worker downloads the same number of buckets. This number is set to 1 .

2. PIR requirement Each PIR item has the same size in bits (padding is allowed):

$$
\forall b_{1}, b_{2} \in L,\left\|b_{1}\right\|=\left\|b_{2}\right\|
$$

This condition comes from the use of PIR.

3. Availability condition For all points in the space of skills, there has to be at least one item containing all tasks matching with this point. In other words, no matter their skills (position in the space), each worker can find a bucket that provides every task they match with.

$A$ bucket $b \in L$ is an item of a packing. We note $|b|$ for the number of tasks contained in the bucket $b$, and $t \in b$ the fact that a task $t$ is included in bucket $b$. The size in bits of a bucket $b$ is denoted as $\|b\|$.

Theorem 6. The use of PIR with libraries which fulfill the packing conditions satisfy $\epsilon_{\kappa}-S I M-C D P$ privacy against coalitions of up to $\tau$ participants.

${ }^{19}$ In general, more files can be downloaded at each worker session, but this does not impact significantly the overall amount of computation and does not impact at all the minimum download size for workers. 
Proof. (sketch) In order to prove the security of packing, we observe that (1) the XPIR protocol has been proven computationally secure in [2, such that it satisfies $\epsilon_{\kappa}$-SIM-CDP, and (2) the use of packing prevents any sensitive information on workers to leak through the number of downloads. Indeed, Condition 71](security) makes each worker call the PIR-get function only once, such that the behaviours of any two workers are indistinguishable. Therefore, the number of PIR-get calls does not depend on profiles. More precisely, the number of PIR-get can only leak information on the number of workers (which is bigger than or equal to the number of PIR-get calls), which does not depend on their profiles, and is already known by the platform.

Before considering how to design an efficient packing scheme, we highlight a few noticeable implications of these conditions. First, due to Condition $7 / 3$ (availability), any worker is matched with at least one bucket. To simplify this model, we propose to focus on a specific kind of packings, that can be seen as a partitioning of the space, where each bucket can be linked to a specific subspace, and where all points are included in at least one of such a subspace. We call partitioned packing such a packing (Definition 8).

Definition 8 (Partitioned Packing). A partitioned packing is a packing that fulfills the following conditions:

1. Each bucket is associated with a subspace of the space of skills.

2. A bucket contains exactly the tasks that intersect with the subspace it is associated with (this means that all workers in this subspace will find at least the task they match with in the bucket)

3. Subspaces associated with the buckets cover the whole space (from Condition 73 (availability)).

4. Subspaces associated with the buckets do not intersect each other

In the following, we will focus on partitioned packing. However, in order not to lose generality, we first prove that these packings do not impact efficiency. Indeed, when it comes to the design of a PIR library, efficiency can be affected by two main issues: the number of items and the size of the largest item (in our case, bucket) impact the communication costs, while the size of the overall library impacts the computation time on the platform. Note that the size of the overall library is equal to the product of the size of items by their number. We show in Theorem 7 that with any packing, we can build a partitioned packing that is equivalent or better.

To prove this theorem, we introduce a specific kind of packing that we call consistent packing, defined in Definition 9. Essentially, a consistent packing is a packing where no useless task is added to any bucket: in all buckets $b$, all tasks match with at least one point (a possible worker profile) which has all her tasks in the bucket $b$. As a result, a consistent packing avoids cases where tasks are in a bucket, but no worker would download it as the bucket does not match with all their needs.

Definition 9 (Consistent packing). A packing $P$ is called consistent if and only if, for all buckets $b \in P$, for all tasks $t \in b$, there exists at least one point $w$ 
in the subspace of $t$ such that all tasks matching with $w$ are in $b$ :

$$
\forall b \in P, \forall t \in b, \exists w,\left(\operatorname{match}(w, t) \wedge \forall t^{\prime} \in T, \operatorname{match}\left(w, t^{\prime}\right) \Rightarrow t^{\prime} \in b\right)
$$

Theorem 7. For any packing $P$ of tasks, there exists a partitioned packing that either has the same size of buckets, number of buckets, or smaller ones.

Proof. (sketch) Let $P$ be a packing of tasks that is not partitioned. To prove that a partitioned packing can be created that is more efficient than $P$, we distinguish two cases. First, we consider that each bucket of $P$ can cover a subspace, containing exactly the tasks that intersect with that subspace (thus fulfilling Conditions 81 and 82 . Then, we prove that any consistent packing (as in Definition 97 fulfills Condition 82 . After that, we consider the case where $P$ does not fulfill this condition, and create a new, smaller packing $P_{f}$ from $P$ that is consistent, and therefore fulfills Condition 82 , and use previous results.

We first consider the case where all buckets of $P$ can cover a subspace while fulfilling Condition 82 , meaning that each bucket contains exactly the tasks that intersect with the subspace it covers. In that case, Condition 811 is trivially fulfilled. If Condition 833 is not fulfilled, this means that there is at least a subspace that is not covered by the packing $P$. Let $w$ be a point in such a subspace. Since $P$ is a packing, the Condition $7 / 3$ (availability) makes it possible to match with any point of the space with at least one bucket. In particular, $w$ can be matched with a bucket $b$. It is enough to extend the subspace associated with $b$ such that it includes $w$ (note that this extension does not break Condition 82 We can proceed that way for any point (or more likely any subspace) that is not covered by a subspace, to associate subspaces to a bucket of $P$, such that this matching fulfills Conditions 81,82 and 83 , If, in this matching, two subspaces associated with buckets of $P$ intersect, it is trivial to reduce one of them to fulfill Condition 84 too. Therefore, if all buckets of $P$ can be matched with a subspace while fulfilling Condition 82 , the theorem holds, since $P$ is equivalent to a partitioned packing.

It can be noticed that if a packing is consistent (Definition 9), Condition 822 is fulfilled. Indeed, if $\forall b \in P, \forall t \in b, \exists w,\left(\operatorname{match}(w, t) \wedge \forall t^{\prime} \in T, \operatorname{match}\left(w, t^{\prime}\right) \Rightarrow t^{\prime} \in\right.$ $b$ ) (Definition 9), then, for all $b$ in $P$, we can take $V$ as the union of the $|b|$ points $w$ described in the equation, one for each task $t$ in $b$. In that case, Condition 82 is fulfilled: for each bucket $b$ and its associated subspace $V$, all tasks in $b$ intersect with $V$ (by definition, as we took $V$ as the union of one point in each task in $b$ ), and $b$ contains all tasks that intersect with $V$ (again, by definition, as each task $t^{\prime}$ that match with a point of $V$ are in $b$ ).

In other words, and using the above case, making a packing consistent is sufficient to create a partitioned packing.

We now consider the case where at least one bucket $b$ of $P$ does not cover a subspace such that Condition 82 does not stand. In particular, $P$ is not consistent. This means that there is at least one task $t \in b$ such that for all points $w$ in the subspace of $t$, there is at least one task $t^{\prime}$ with which $w$ matches and that is not contained by the bucket $b$. In other words, no points in $t$ can be matched with the bucket $b$, as $b$ lacks at least one task for each point of $t$. As a consequence of 
the Condition 733 (availability), this means that all points in $t$ are matched with another bucket. Therefore, the task $t$ can be removed from bucket $b$, without breaking the properties of a packing, and without increasing the number of buckets, the minimal size of buckets. We proceed so, by removing all such tasks in all buckets recursively: this trivially ends thanks to the finite number of tasks and buckets. By construction, the final packing $P_{f}$ is consistent.

Therefore, packing $P_{f}$ is smaller than $P$, and fulfills Condition 82 , and we proved in the first case that a packing that fulfills this condition is equivalent to a partitioned packing, so $P_{f}$ is equivalent to a partitioned packing.

\subsection{Optimizing the packing}

With this secure partitioned packing approach, we can discuss how to optimize the overall complexity. First, it can be noticed that Conditions 722 and 7/3 (PIR requirement and availability) set a minimal size of bucket: according to Condition 73 (availability), there has to be a bucket containing the largest (in bits) intersection of tasks, and Condition 72 (PIR requirement) prevents any bucket from being smaller. Furthermore, this minimum is reachable if we consider a packing that creates a partition for each different intersection of tasks and pad to the largest one. However, by building a different bucket for all the possible intersections of tasks, this packing strategy is likely to lead to a very large number of buckets (e.g. if a task's subspace is included in another, this packing leads to two buckets instead of one: one containing both tasks, and the other containing only the largest one as it is a different intersection), while we would like to minimize it (and not only the size of buckets). Therefore, although this packing scheme reaches the minimal size of buckets, we cannot consider it as optimal. However, it illustrates what we call an acceptable packing (Definition 10, which will be used to define optimality: a packing in which the size of buckets is minimal.

\section{Definition 10 (Acceptable partitioned packing).}

Let $E$ be a multi-dimensional space, $T$ a set of tasks, i.e. a set of positively weighted hyper-rectangles (the hyper-rectangle is the volume of the task, and the weight is their size in bits, denoted $w_{t}$ for $t \in T$ ) in the space $E$ and $P$ a packing of these tasks. We call weight of a packing $w_{P}$ the size in bits of a bucket in $P$ (due to Condition 72 (PIR requirement), this size is unique). We call weight of a point $w_{p}$ in $E$ the sum of the weights of all tasks in $T$ which match with $p$.

We call minimal weight $m_{T}$ of the set of tasks $T$ the maximum weight of a point in $E$ : it is the maximum size a worker could require to download. A partitioning $P$ is called acceptable for $T$ if the size of $P$ is equal to $m_{T}: m_{T}=w_{P}$.

NP-hardness of optimal packing To define optimality, we take this minimum size of buckets, but also try to minimize the number of buckets (or in an equivalent way, the size of the PIR library), as expressed in Definition 11.

Definition 11 (Optimal partitioned packing). For a set of tasks T, we call optimal packing an acceptable packing that minimizes the number of buckets. 
However, we prove in Theorem 8 that determining whether there exists an acceptable packing of size $n$ is NP-hard, and therefore, finding the optimal partitioned packing is also NP-hard.

Theorem 8. Given a set of tasks $T$, it is NP-hard in $|T|$ to determine whether there exists an acceptable partitioning of $n$ buckets. We call $\mathcal{P}(T, n)$ this problem.

Proof. (sketch) To prove that this problem is NP-hard, it is enough to demonstrate that a certain problem $\mathcal{P}^{+}$known to be NP-complete can be polynomially reduced to $\mathcal{P}$.

We recall that the Partition Problem is NP-complete (see [21]): $\mathcal{P}^{+}(S)$ : given a multiset $S$ of $N$ positive integers $n_{i}, i \in[0, N-1]$, decide whether this multiset can be divided into two submultisets $S_{1}$ and $S_{2}$ such that the sum of the numbers in $S_{1}$ equals the sum of the numbers in $S_{2}$, and the union of $S_{1}$ and $S_{2}$ is included in $S$.

Let us consider a multiset $S$ and the problem $\mathcal{P}^{+}(S)$. We assume the existence of a deterministic algorithm $A$ that solves $\mathcal{P}(T, n)$ in a polynomial time in $|T|$. We first distinguish a trivial case where the problem $\mathcal{P}^{+}(S)$ can be solved in polynomial time. Then, we build an algorithm that uses $\mathcal{P}(T, 3)$ to solve $\mathcal{P}^{+}(S)$ in polynomial time similarly to the remaining cases, which leads to a contradiction.

We first consider a trivial case: if there exists $n_{k}$ in $S$ such that $n_{k}>$ $\sum_{i \in[0, N-1], i \neq k} n_{i}$, then we return False. Deciding whether $S$ falls in that specific case is linear in $|S|$, and so is the computation of the answer. If not, let $E$ be a one dimensional space, with bounds $[0,|S|+1[$. We build $T$ as a set of $|S|+1$ tasks $\left(T=\left\{t_{i}, i \in[0, N]\right\}\right)$, such that no task intersects with each other: therefore, the minimum size $m_{T}$ of $T$ (from Definition 10 ) will be the same as the size of the biggest task $t$ in $T$. The $|S|$ first tasks are all associated with an element of $S$, while the last one will be used to fix $m_{T}$. More precisely, we build $T$ as follows:

-- the range of $t_{i}$ is $[i, i+1[$

-- for $i \neq N$, the weight of $t_{i}$ is equal to the value of $n_{i} ; w_{t_{N}}=\frac{\sum_{i \in[0, N-1]} n_{i}}{2}$.

Building $T$ and $t_{\max }$ is subpolynomial.

By hypothesis, $\forall k, n_{k} \leq \sum_{i \in[0, N-1], i \neq k} n_{i}$ (as we dealt with this case previously), and by construction, no hyper-rectangle intersects any other, so the minimal weight is the size of the biggest task, which is the last one: $m_{T}=$ $\max _{t_{i}}\left(w_{t_{i}}\right)=w_{t_{\max }}$. Therefore, if $S$ can be divided into two submultisets $S_{1}$ and $S_{2}$ of the same size, this size is $\frac{\sum_{i \in[0, N-1]} n_{i}}{2}$, and $\mathcal{P}(T, 3)$ answers True.

Reciprocally, if $\mathcal{P}(T, 3)$ answers True, this means that there exists a packing of size 3 such that no packing is bigger than $m_{T}=\frac{\sum_{i \in[0, N-1]} n_{i}}{2}$. In particular, as $w_{t_{N}}=m_{T}$, this means that no task is added to the bucket containing it, and that the two remaining buckets contain all tasks $t_{i}, i \neq N$. If one of these buckets where smaller than $m_{T}$, the other would be bigger than $m_{T}\left(\right.$ as $\left.m_{T}=\frac{\sum_{i \in[0, N-1]} n_{i}}{2}\right)$, and therefore, both buckets weight exactly $m_{T}$. Therefore, it is possible to separate $S$ in $S_{1}$ and $S_{2}$ such that the sum of the numbers in $S_{1}$ equals the sum of the $S_{2}$ 
by taking all the elements corresponding to the tasks in the first bucket for $S_{1}$, and the elements corresponding to the second bucket for $S_{2}$.

Therefore, if we are not in the trivial case treated above, $\mathcal{P}(T, 3)$ answers True if and only if $\mathcal{P}^{+}(S)$ is true in polynomial time. As both deciding whether we are in that trivial case and computing the answer in that trivial case can be computed in polynomial time, an algorithm deciding $\mathcal{P}^{+}(S)$ in polynomial time can be built. The assumption of $\mathcal{P}(T, n)$ not being NP-hard leads to a polynomial algorithm solving $\mathcal{P}^{+}$, which is absurd, so $\mathcal{P}(T, n)$ is NP-hard.

Static packings Another point can be highlighted: the difference between what we call static packing and dynamic partitioning. Indeed, when trying to optimize the use of partitioned buckets, two main approaches can be used: adapt buckets to tasks, or adapt tasks to buckets. In the first case, we consider a fixed set of tasks, and try to build partitions in order to minimize the cost of PIR. On the one hand, this optimization makes it possible to perform the best with any set of tasks. On the other hand, as we consider a fixed set of tasks, we may have to compute a new partitioning when this set evolves (when a task is added or removed, at least when it affects the largest bucket). In the second case however, we consider a fixed partitioning, that is independent from the set of tasks. This method is more likely to be suboptimal, but it avoids heavy computation of optimal packing and allows a greater flexibility in the context of crowdsourcing, by allowing a large variety of choices and policies from the platform, which can even lead to other kinds of optimization. For instance, it allows the platform to manage prices policies (e.g. making tasks pay for each targeted subspace, higher prices for tasks willing to target highly demanded subspaces, etc.), in order to even the load within the whole space, and to reduce the redundancy of tasks within the PIR library (tasks that target more than one partition).

As finding the optimal is NP-hard, we prefer to set aside dynamic packings, as its main asset is the theoretical possibility to reach optimality while remaining unrealistic in a real-life scenario, and focus instead on static packings.

Static packing means that the design of partitions is independent of tasks: the tasks contained within the bucket may change, but not the subspace delimited by the partition. These heuristic packing schemes are not optimal in general but may be affordable in real-life scenarios. We propose to use a simple heuristic static packing scheme, the PKD PIR Packing, consisting in using the partitioned space of workers profiles computed primarily for task design purposes: to each leaf partition corresponds a bucket containing all the tasks that have metadata intersecting with it (possibly with padding). The resulting algorithm is presented in Algorithm 3. The accordance of this scheme with the distribution of workers can lead to both useful and efficient buckets (as assessed experimentally, see Section 5), and the stability over time of the space partitioning (static approach) makes it easier to design policies to approach optimality through incentives on the task design (rather than through the bucket design). 


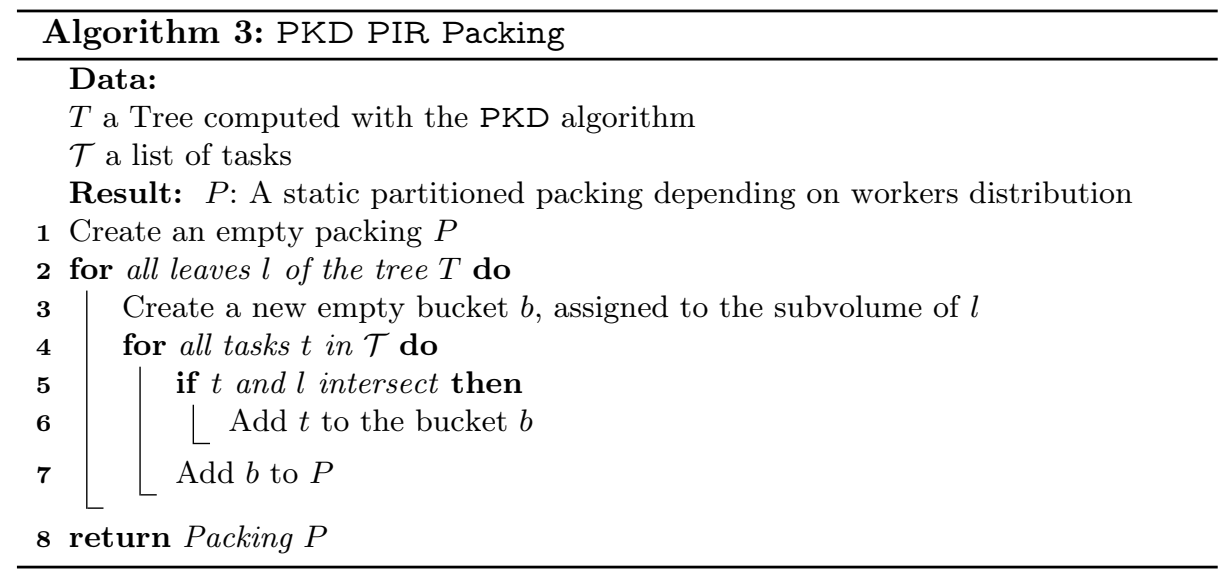

\section{Experimental Validation}

We performed a thorough experimental evaluation of the quality and performances of both the PKD algorithm and our PKD PIR Packing heuristic (that we abbreviate as PIR in the experiments).

\subsection{Datasets}

In this section, we introduce the datasets and data generators that are used in our experiments.

Realistic Dataset. To the best of our knowledge there does not exist any reference dataset of worker profiles that we could use for our experiments. This led us to building our own dataset from public open data. The StackExchang ${ }^{20}$ data dumps are well-known in works related to experts finding. We decided to use them as well in order to perform experiments on realistic skills profiles. We computed profiles by extracting skills from users' posts and votes. In StackExchange, users submit posts (questions or answers) that are tagged with descriptive keywords (e.g., "python programming") and vote positively (resp. negatively) for the good (resp. bad) answers. We consider then that each user is a worker, that each tag is a skill, and that the level of expertise of a given user on a given skill is reflected on the votes. We favored a simple approach for computing the expertise of users. First, for each post, we compute a popularity ratio as follows: $r$ = upvotes/(upvotes + downvotes), where upvotes is the number of positive votes of the post and downvotes is the number of negative votes. Second, for each user $p_{i}$, for each tag $j$, the aggregate level of expertise $p_{i}[j]$ is simply the average popularity ratio of the posts from $p$ tagged by $j$. Note

${ }_{20}$ StackExchange is a set of online forums where users post questions and answers, and vote for good answers https://archive.org/download/stackexchange 
that more elaborate approaches can be used (see the survey [35]). Finally, we removed the workers that do not have any skill level higher than 0 . We applied this method on three StackExchange datasets: stackoverflow.com-Posts.7z, stackoverflow.com-Tags.7z, and stackoverflow.com-Votes.7z which resulted in $1.3 \mathrm{M}$ worker profiles ${ }^{21}$. Figure 3 (a) shows for ten common skills ${ }^{22}$ and for the possible levels divided in ten ranges (i.e., $[0.0,0.1[,[0.1,0.2[, \ldots,[0.9,1])$ their corresponding frequencies. It shows essentially that whatever the skill considered, most workers have a skill level at 0 . The rest of the distribution is not visible on this graph so we show in Figure 3 (b) the same graph but excluding, for each tag, the workers having a skill level at 0 .

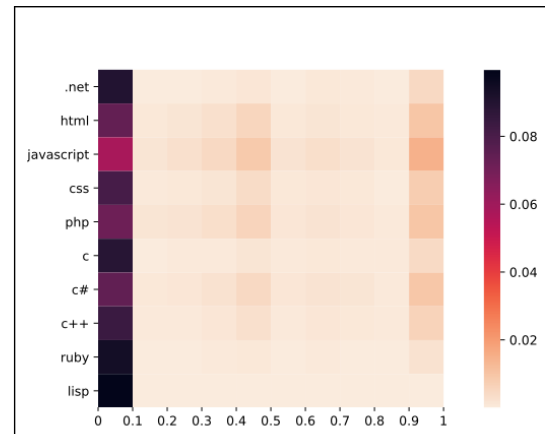

a) On the x-axis: Skill level. On the y-axis: Skill. On the heatmap: frequency of the given skill-level on the given skill within workers - including the workers having a skill level at 0 .

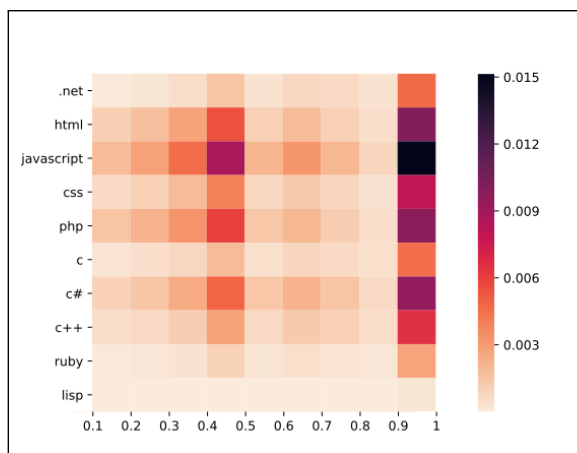

b) On the x-axis: Skill level. On the y-axis: Skill. On the heatmap: frequency of the given skill-level on the given skill within workers - excluding the workers having a skill level at 0 .

Fig. 3. Frequencies of ten common skills within the STACK dataset.

Data Generators. We performed our experiments over both synthetic and realistic data. Our two synthetic generators are specifically dedicated to evaluating the PKD algorithm with two different kinds of assumptions. First, our UNIF synthetic data generator draws skills uniformly at random between 0 and 1 (included) (1) for each dimension of a worker's profile and (2) for each dimension of a task (more precisely, a min value and a max value per dimension). Second, our ONESPE generator considers that workers are skilled over a single dimension and that tasks look for workers over a single dimension. The specialty of each worker is chosen uniformly at random, and its value is drawn uniformly at random between 0.5 and 1 . The other skills are drawn uniformly at random between 0 and 0.5. Similarly to workers, the specialty looked for by a task is chosen uniformly at

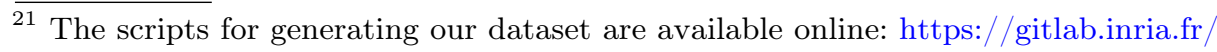
crowdguard-public/data/workers-stackoverflow

${ }^{22}$ The ten common skills considered are the following: .net, html, javascript, css, php, c, c\#, c++, ruby, lisp. 
random as well, its min value is chosen uniformly at random between 0.5 and 1 and its max value is set to 1 . The min values of the other dimensions of a task are 0 , and their max values are chosen uniformly at random between 0 and 0.5 . Although this second heuristic is obviously not perfect, it seems far more realistic than the previous one. For the two task generation heuristics, we require that all tasks must contain at least one worker so that the mean error can be correctly computed.

Finally, our realistic data generator, called STACK, consists in sampling randomly workers (by default with a uniform probability) from the STACK dataset. For our experiments, we generated through STACK workers uniformly at random and performed the ONESPE task generation strategy described above.

\subsection{PKD algorithm}

Quality of the PKD algorithm. For our experiments, we implemented the PKD algorithm in Python 3 and run our experimental evaluation on commodity hardware (Linux OS, 8GB RAM, dual core $2.4 \mathrm{GHz}$ ). In our experiments, each measure is performed 5 times (the bars in our graphs stand for confidence interval), $1 k$ tasks, 10 dimensions, and $\tau=1$.

In Fig. 4 (a), we fix the privacy budget to $\epsilon=0.1$, the number of bins to 10 , and the number of workers to $10 k$, and we study the impact of the depth of the tree on the quality. UNIF achieves the lowest error, as long as the tree is not too deep. This can be explained by the uniform distribution used in the generation method, which matches the uniform assumption within leaves in the tree. When the depth (and the number of leaves) grows, this assumption matters less and less. ONESPE is more challenging for the PKD algorithm because it is biased towards a single skill. It achieves a higher error but seems to benefit from deeper trees. Indeed, deep trees may be helpful in spotting more accurately the specialized worker targeted. The results for STACK are very similar. For all of these distributions, we can see that having a tree deeper than the number of dimensions leads to a significant loss in quality.

In Fig. 4 (b), we analyze the variations of quality according to the value of $\epsilon$, with 10 bins, a depth of 10 , and $10 k$ workers. In this case, the relative error seems to converge to a non-zero minimum when $\epsilon$ grows, probably due to inherent limits of KD-Tree's precision for tasks.

In Fig. 4 (c), we fix the privacy budget to $\epsilon=0.1$, the depth of the tree to 10 and $10 k$ workers. We can see the impact of the number of bins for each histogram used to compute a secure median. This value does not greatly impact the relative error for the UNIF and STACK models, although we can see that performing with 1 bin seems to give slightly less interesting results, as it looses its adaptability toward distributions. For the ONESPE model, having only 1 bin gives better results: indeed, the uniformity assumption within the bin implies that all dimensions are cut at 0.5 , which is also by construction the most important value to classify workers generated with this procedure.

In Fig. 4 (d), we compare the quality according to the number of workers with $\epsilon=0.1,10$ bins and a depths of 10 . As the $\epsilon$ budget is the same, the noise is 
independent from this number, and thus, the quality increases with the number of workers.

We can notice that our results for the relative error are quite close to the state of the art results, such as the experiments from [9], which are performed on 2-dimensional spaces only, with strong restrictions on the shapes of queries (tasks in our context) and in a centralized context.

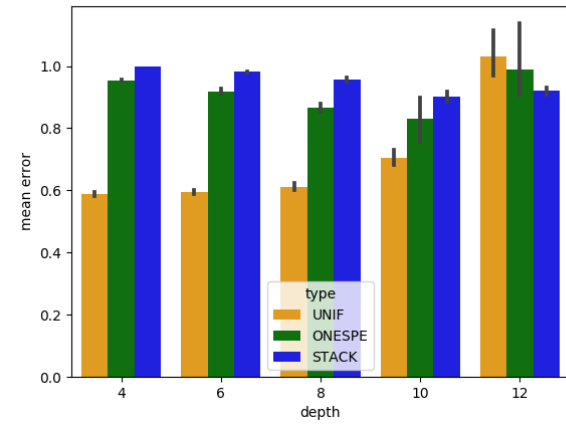

a) Variations according to the depth of the tree.

10 dimensions, $10 k$ workers, $1 k$ tasks, $\tau=1, \epsilon=0.1,10$ bins

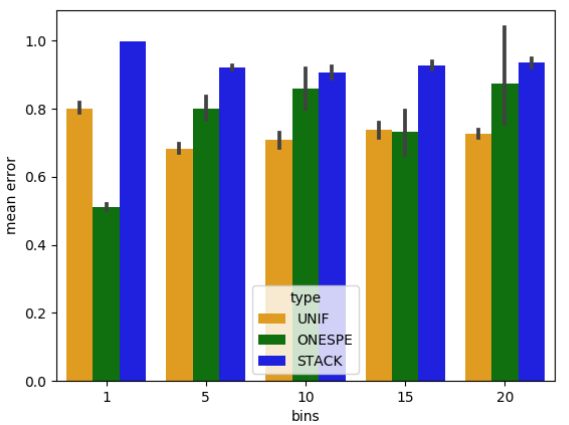

c) Variations according to the number of bins.

10 dimensions, $10 k$ workers, $1 k$ tasks, $\tau=1, \epsilon=0.1$, depth $=10$

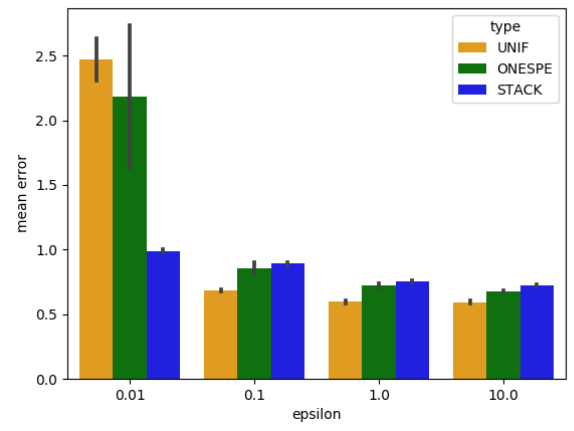

b) Variations according to $\epsilon$ privacy budget.

10 dimensions, $10 k$ workers, $1 k$ tasks, $\tau=1,10$ bins, depth $=10$

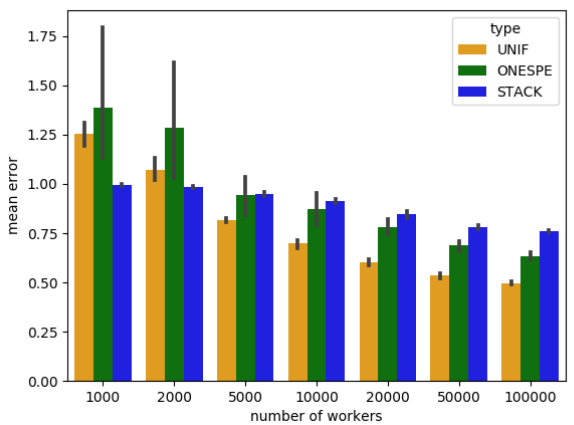

d) Variations according to the number of workers.

10 dimensions, $1 k$ tasks, $\tau=1, \epsilon=0.1,10$ bins, depth $=10$

Fig. 4. Mean relative error (see Definition 5 , the lower the better)

Computation time of the PKD algorithm. Our performance experiments were performed on a laptop running Linux OS, equipped with $16 G B$ of RAM and an Intel Core $i 7-7600 U$ processor. We measured the average computation time across 100 experiments of each of the atomic operations used in the PKD 
algorithm: encryption, partial decryption, and encrypted addition. The results are summed up in Fig. 5. with keys of size 2048 bits, using the University of Texas at Dallas implementation for its accessibility 23 . We use our cost analysis together with these atomic measures for estimating the global cost of the PKD algorithm over large populations of workers (see Equation 4. Equation 5 , and Equation 6 in Section 3.3 .

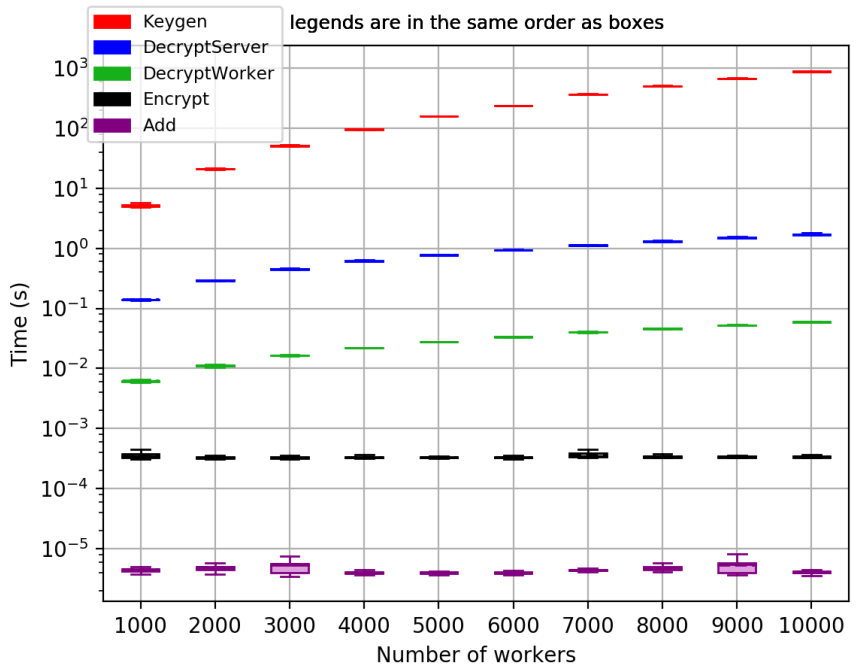

Fig. 5. Computation time of homomorphically encrypted operations

We can observe that the slowest operation is by far the generation of the keys. However, since this operation is performed only once, the cost of less than 1000 seconds (about 17 minutes) for $10 k$ workers is very reasonable: this operation can be performed as soon as there are enough subscriptions, and the keys may be distributed whenever the workers connect. The other operations are faster individually, but they are also performed more often. For $10 k$ workers, 10 workers required for decryption, a depth of the KD-Tree of 10 and 10 bins, we can observe that: each worker will spend less than 10 seconds performing encryptions, the platform will spend less than 1000 seconds performing encrypted additions, the average worker will spend less than 1 second performing decryptions, and the platform will spend less than 3000 seconds performing decryptions.

Overall, these costs are quite light on the worker side: less than 20 seconds with commodity hardware. On the server side, the computation is more expensive (about one hour), but we could expect a server to run on a machine more powerful than the one we used in our experiments. Additionally, it is worth to note that: (1) the perturbed skills distribution is computed only once for a given a population of workers and then used repeatedly, and (2) we do not have any

23 http://cs.utdallas.edu/dspl/cgi-bin/pailliertoolbox/index.php?go=download 
real time constraints so that the PKD algorithm can run in background in an opportunistic manner.

\subsection{Assignment using packing}

Quality of our packing. We here propose to evaluate the quality of our partitioned packing approach. Our experiments are performed with the same settings as those used to measure the quality of the PKD algorithm (see Section 5.2). To do so, we propose two main metrics. First, me measure the mean precision for tasks, as defined in Definition 6. Although this measure is useful to understand the overall improvement of our approach, it does not take into account the fact that downloads caused by PIR scale with the largest item. Therefore, we introduce a second measure, the mean number of tasks that a worker would download. This value, that we call maximum tasks, is computed as the maximum number of tasks that a leaf of the KD-tree intersects with: indeed, due to Condition 72 (PIR requirement), all workers will download as many data as contained in the biggest bucket.

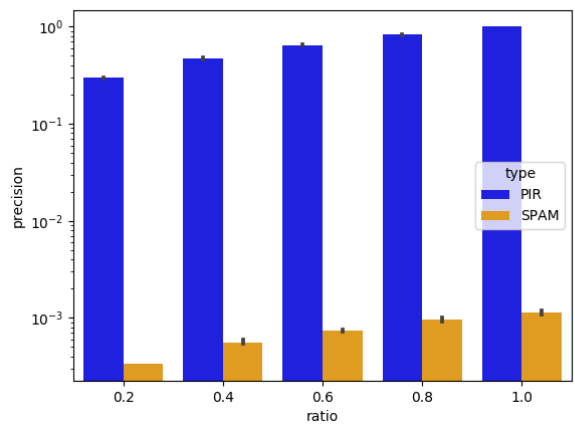

a) Precision in log scale, according to the ratio of leaf taken by task for the UNIF model.

10 dimensions, $10 k$ workers, $1 k$ tasks, $\tau=1, \epsilon=0.1,10$ bins, depth $=10$

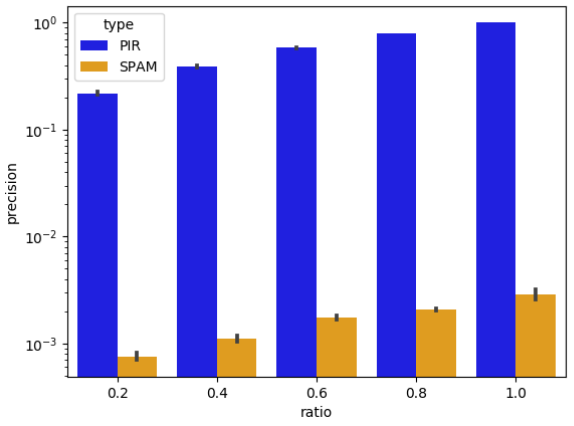

b) Precision in log scale, according to the ratio of leaf taken by task for the ONESPE model.

10 dimensions, $10 k$ workers, $1 k$ tasks, $\tau=1, \epsilon=0.1,10$ bins, depth $=10$

Fig. 6. Precision (the higher the better)

In the task generation methods introduced previously, tasks are built independently from the KD-tree itself. This independence was logical to measure the quality of the PKD algorithm. However, this very independence leads to poor results when it comes to building efficient packing on top of a KD-tree: as tasks are independent from the KD-tree, they have little restriction on how small they are (meaning that few workers will match with them, although all workers in leaf that intersect with it will download it), or on how many leaves they intersect with, leading to low precision, and high size of buckets.

Therefore, we introduce a new method to build tasks: SUBVOLUME. With this method, we build tasks as subleaves, meaning that all tasks are strictly 
included within one leaf of the KD-tree. Furthermore, we also enforce the size of the task as a parameter, such that the volume of the task is equal to a given ratio of the task. More precisely, for a ratio $r \in[0,1]$, a space $E$ of $d$ dimensions and a picked leaf $l$, the interval of a task in a given dimension $d_{i} l_{d_{i}} \times r^{1 / d}$, where $l_{d_{i}}$ is the interval of the leaf in dimension $d_{i}$. The SUBVOLUME model of tasks can easily be introduced by economic incentives from the platform, such as having requesters pay for each targeted leaf, which is likely to induce a maximization of the volume taken, and a reduction of the tasks that intersect with more than one leaf. Note that we do not perform experiments with this generation of tasks on the Stack dataset, as most workers have their skills set to either 0 or 1, which leads to very unreliable results as tasks almost never encompass either of these values.

The comparison between the PKD PIR Packing heuristic and the spamming approach using this new method to generate tasks, presented in Figure 6, show that our approach improves precision by at least two orders of magnitude. Also, note that for $r=1$, the precision is equal to 1 in the PIR approach. This result comes from the fact that, with $r=1$, all workers within a leaf are targeted by all tasks that intersect with that leaf, meaning that they do not download irrelevant tasks.

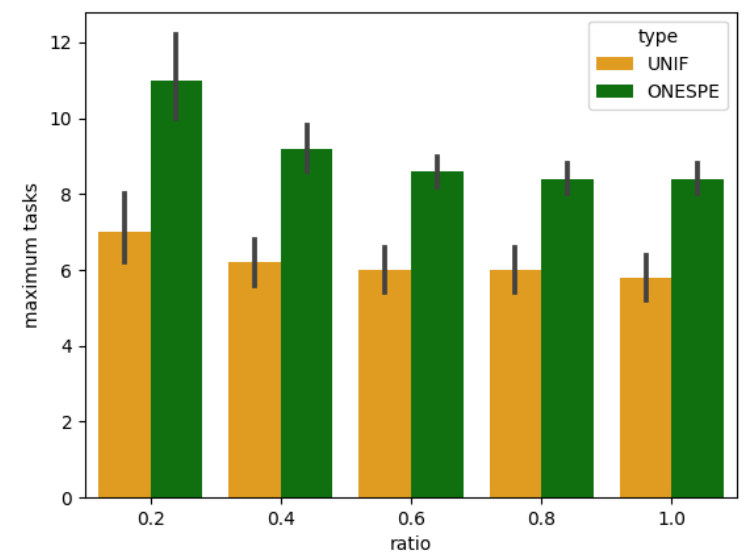

Fig. 7. Number of tasks downloaded according to the ratio of leaf taken by task for the packing approach. 10 dimensions, $10 k$ workers, $1 k$ tasks, $\tau=1, \epsilon=0.1,10$ bins, depth $=10$

The maximum number of tasks connected to a leaf, showed in Figure 7 , show that the cost of download is also significantly improved (these values are to be compared to 1000 , the total number of tasks that are downloaded with the spamming approach) also shows great improvement (around 2 orders of magnitude), as tasks are more evenly spread within the leaves (there are $2^{10}=1024$ leaves for a depth 10 of the tree, which can explain this improvement).

Cost of the PIR protocol. We here study the impact of the number of files and of the size of files on the computation time. In the experiments, we used a computer 
with $8 G B$ of RAM, and a Ryzen 51700 processor, using the implementation of $[2]^{24}$.

As we can see in Figure 8 with keys of size 1024 bits, computation time is proportional to the overall size of the PIR library (the coefficient of determination gives $\left.r^{2}=0.9963\right)$, and that it grows at $0.14 s / M B$ for a given request, as long as the library can be stored in RAM ${ }^{25}$.

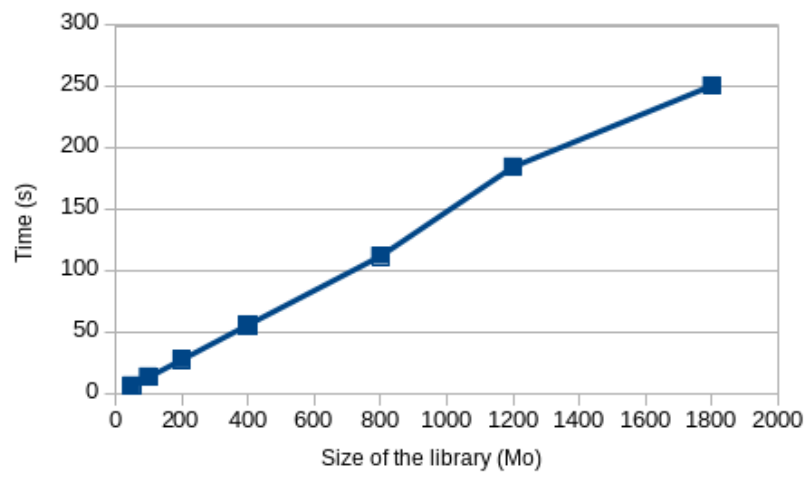

Fig. 8. Computation time of the retrieval of a file according to the PIR library's size

We now evaluate the maximum number of tasks $n_{\max }$ that our system can take into account, according to two parameters: first, the time $t$ that workers accept to wait before the download begins, and second, the size $s$ that workers accept to download. As $n_{\max }$ does not solely depend on $t$ and $s$, we introduce a few other notations:

-- $f$ is the expansion factor of the encryption scheme.

-- $\mid$ task $\mid$ the mean size of a task.

-- $k$ the proportion of tasks that are in the biggest leaf of the KD-tree (for instance, $k=0.1$ means that the biggest leaf contains one tenth of all tasks)

-- depth, the depth of the KD-tree (that is linked with the number of buckets)

In the spamming approach, the maximum number of tasks that can be managed by our system is independent from $t$ and can be simply computed as:

$$
n_{\max , S P A M}=\frac{s}{|t a s k|}
$$

For the PKD PIR Packing heuristic, both $s$ and $t$ lead to a limitation on $n_{\max , P I R}$. We first consider the limit on the computation time $t$ : according to our results in Figure 8 , the PIR library cannot be bigger than $\frac{t}{0.14}$, and the size of a bucket, can be computed as $k \times|t a s k| \times n_{\max , P I R}$ (by definition

24 https://github.com/XPIR-team/XPIR

25 If it cannot, accesses to the secondary storage device are necessary. This would increase the runtime accordingly. However, since the library is scanned once per query, sequentially, the cost would remain linear in the size of the library. 
of $k$, as all buckets weight as much as the biggest one). As the library can be computed as the product of the number of buckets and their size, this leads us to $2^{\text {depth }} \times k \times|t a s k| \times n_{\max , P I R} \leq \frac{t}{0.14}$, or equivalently $n_{\max , P I R} \leq$ $\frac{t}{0.14 \times 2^{\text {depth }} \times k \times|t a s k|}$. We now consider the limit $s$ on the size of download. For each worker, the size of a download will be the same, computed as the product of the expansion factor and the size of a bucket: $f \times k \times n_{\max , P I R} \times|t a s k| \leq s$. This inequality leads to $n_{\max , P I R} \leq \frac{s}{f \times|t a s k| \times k}$. By combining these two inequalities, $n_{\max , P I R}$ takes its maximum value when

$$
n_{\max , \text { PIR }}=\min \left(\frac{s}{f \times \mid \text { task } \mid \times k}, \frac{t}{2^{\text {depth }} \times 0.14 \times \mid \text { task } \mid \times k}\right)
$$

In Figure 9, we compare the number of tasks that a crowdsourcing platform can manage with different values of $t$ and $s$, using either the spamming approach or our PKD PIR Packing heuristic. For the sake of simplicity, we consider that the expansion factor $f$ is 10 , although smaller values are reachable with XPIR protocol [2]. This factor will impact the amount of tasks that a worker can download. We take $d=10$ similarly to our previous experiments. We consider a mean size of task $|t a s k|=1 M B$. It can be noticed that $|t a s k|$ has no impact on the comparison $\left(\frac{\max _{n, P I R}}{\max _{n, S P A M}}\right.$ does not depend on $\left.|t a s k|\right)$. For $k$, we consider two possible values: $k=0.01$, as suggested by the experiments in Figure 7 , and $k=\frac{1}{2^{10}}$, which represents the optimal case, where tasks are perfectly spread among buckets (for instance, due to strong incentives from the platform).

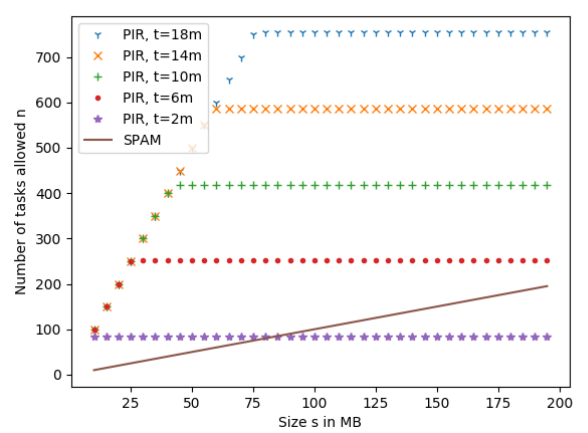

a) Number of tasks $n$ manageable by our system according to the size $s$ a worker accepts to download.

$$
\begin{gathered}
k=0.01, \mid \text { task } \mid=1 M B, f=10, \\
\text { depth }=10
\end{gathered}
$$

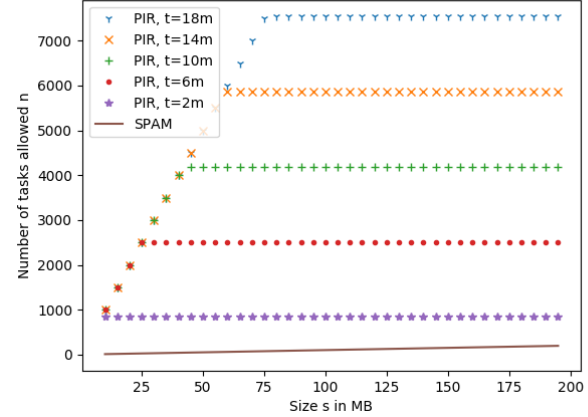

b) Number of tasks $n$ manageable by our system according to the size $s$ a worker accepts to download.

$$
\begin{aligned}
k=0.001, \mid \text { task } \mid & =1 M B, f=10, \\
\text { depth } & =10
\end{aligned}
$$

Fig. 9. Precision (the higher the better) ; curves are in the same order as the captions

In these experiments, we can notice that our approach depends on both the computation time allowed and the size of the number of task in the largest bucket. In a real-life scenario, platforms would benefit from enforcing incentives 
to even the load between buckets. However, if workers are willing to limit their download to less than $100 M B$, the PKD PIR Packing heuristic outperforms the spamming approach as long as users are willing to limit their download even with relatively short computation times (less than 10 minutes) by up to several orders of magnitude. Our method is especially interesting in settings where the bandwidth is low (e.g. with mobile devices), with low values of $s$. On the opposite, it is interesting to highlight that high computation times are not necessarily prohibitive: as the computation is performed by the platform, a worker could very well ask for a bucket of tasks and download it later on when it is ready.

\section{Discussion}

In this section, we propose a discussion on questions raised by our work that are not our primary focus. More precisely, we elaborate our views on updates that our system may or may not allow (both for the PKD algorithm and the PKD PIR Packing heuristic), with some advantages and drawbacks.

\subsection{Updating tasks and PIR libraries}

In this work, we dealt with the download of tasks as a one-shot download, meaning that a worker will download tasks once and for all. However, in a real-life scenario tasks are likely to evolve (e.g. new tasks will be added and old tasks will be outdated), and workers are equally likely to update their tasks. Without further improvement, our design would require each worker to download a whole packing for each update of the available tasks. However, more elaborate approaches are possible. Although it is not our focus to develop them exhaustively, we propose a few tracks that are likely to diminish the costs greatly.

For that purpose, we propose to divide time into fixed duration periods (e.g. a day, a week, etc.) and to additionally take into account the period at which a task is issued in order to pack it. We give below two options for allowing updates. Although their improvement have not been quantified nor validated experimentally, These schemes aim at increasing the memory cost on the server in order to alleviate the overall computation required.

Packing by Period A simple scheme that allows easier updates while reducing the size of single PIR request consists in designing packing not only according to a specific partitioning but also according to time periods. The platform builds one PIR library per period, i.e., considering only the tasks received during that period ${ }^{26}$. Workers simply need to perform PIR requests over the missing period(s)

${ }^{26}$ In this kind of methods, a task can be either maintained into its starting period up till it's lifespan, or one can consider keeping up a limited number of periods (e.g. all daily periods for the current month) and re-adding tasks on new periods packing each time they are deleted (e.g. for tasks that are meant to be longer than a month). More elaborate or intermediate methods are also possible, but we will not explore this compromise in this paper. 
(one request per missing period). As a result, the PIR-get function is executed on the library of the requested period, which is smaller than or equal to the initial library.

However, this scheme may result in high costs if the distribution of tasks is skewed. For instance, let's consider two time periods $p_{1}$ and $p_{2}$, two subspaces of the space of skills $s_{1}$ and $s_{2}$, and three tasks $t_{1}, t_{2}$ and $t_{3}$ such that $t_{1}$ and $t_{2}$ appear only in $p_{1}$ and $s_{1}$, while $t_{3}$ appears only in $p_{2}$ and $s_{2}$. In that case, all workers will download first the PIR item for period $p_{1}$, which is the same size as $w_{t_{1}}+w_{t_{2}}$ (due to padding for workers not in $p_{1}$ ) and then a second PIR item for $p_{2}$, of size $w_{t_{3}}$. Without that period strategy, a worker who performs regular updates would have downloaded tasks $t_{1}$ and $t_{2}$ (or equivalent size) twice due to the update, and $t_{3}$ once, but a worker who would not have performed the intermediary download would have downloaded $\max \left(w_{t_{3}}, w_{t_{1}}+w_{t_{2}}\right)$. Therefore workers who update frequently would benefit from this strategy, while workers who do not would have worse results.

Personalized Packing by Period In order to tackle the previously mentioned issue caused by skewed distribution of tasks, and to optimize the size of the downloaded bucket for any frequency of downloads, we propose to adapt the packing to the workers frequency of downloads.

Indeed, we observe that it is enough to perform as many packings as there are possible time-lapses for workers, e.g., one packing for the last period, one packing for the last two periods, one packing for the last three periods, etc.. As a result, each PIR-get request is associated with a time-lapse in order to let the PIR server compute the buckets to be downloaded (or use pre-computed buckets). With this method, we can get the best of both worlds with the previous example: someone who downloads frequently will only have small updates, while someone who does not will not suffer from overcosts.

The main (and limited) drawback of this method is that the platform will have to store multiple PIR-libraries, which increases the storage required.

Security of Packing by Period In both of the above schemes, we consider multiple downloads from workers. Even worse, in the second case the number of downloads may vary depending on workers habits. If the above proposition were to be used, more accurate proofs of security would have to be done. Although it is not our focus to propose them in this article, we provide here some intuitions on their requirements. In the first case, the number of downloads is the same for all workers, and would therefore not lead to great modifications of our proof. In the second case however, the number of downloads depends on the frequency of downloads of workers. In order not to reveal information about worker's profiles, a new hypothesis is likely to be required, that states or implies that the frequency of downloads of workers is independent from their profiles. 


\subsection{Updating PKD}

The PKD algorithm is not meant to allow users to update their profiles, as they would have to communicate information to do it, and this would either break our security policy, or exceed the $\epsilon$ privacy budget. However, departures or arrivals are not inherently forbidden by our security policy. A simple and naive way to upgrade the PKD algorithm to take new arrivals into account is to create multiple KD-trees, and to combine them. For instance, one could imagine using the PKD algorithm on every new $k$ arrivals (e.g. $k=1000$ or $k=10000$ ). The estimation of workers within a subspace would be the sum of the estimations for each KD-tree, and a new PIR library could be built for each of these KD-trees. For retrieval of workers, as it is impossible to know where the worker was, the most naive way to proceed is to retrieve a given value to each leaf of the approximated KD-tree, for instance $\frac{n_{\text {leaf }}}{n_{\text {tre }}}$, where $n_{\text {leaf }}$ is the approximated number of workers in the leaf, and $n_{\text {tree }}$ the total number of workers. Once again, more elaborate methods are possible, but stand out of the focus of this paper.

\section{Related Work}

Privacy-Preserving Task Assignment. Recent works have focused on the privacypreserving task assignment problem in general crowdsourcing. In [5], each worker profile - a vector of bits - is perturbed locally by the corresponding worker, based on a local differentially private bit flipping scheme. A classical task-assignment algorithm can then be launched on the perturbed profiles and the tasks. An alternative approach to privacy-preserving task-assignment has been proposed in [20]. It is based on the extensive use of additively-homomorphic encryption, so it does not suffer from any information loss, but this has a prohibitive cost in terms of performance. Other works have focused on the specific context of spatial crowdsourcing [38/40/37. They essentially differ from the former in that spatial crowdsourcing focuses on a small number of dimensions (typically, the two dimensions of a geolocation) and is often incompatible with static worker profiles. All these works explore solutions to ensure an assignment between tasks and workers in a private way, and are complementary to our approach.

Decentralized Privacy-Preserving Crowdsourcing Platform. ZebraLancer [28] is a decentralized crowdsourcing platform based on blockchains, zero-knowledge proofs, and smart contracts and focuses on the integrity of the reward policies and the privacy of the submissions of workers against malicious workers or requesters (e.g., spammers, free-riders). Zebralancer does not consider using worker profiles (neither primary nor secondary usages).

Privacy-Preserving KD-Trees. The creation and the publication of private KDTrees has been studied in depth in [9], but in our context, this work suffers from two main defficiencies. First, it considers a trusted third party in charge of performing all the computations while in our work we do not assume any trusted third party. Second, it restricts the number of dimensions to two, which 
is unrealistic in our high-skills crowdsourcing context. Enhancements to the technique have been proposed, for example [33, but without tackling the trusted third party assumption.

Privacy-Preserving COUNTs. Other differentially private count algorithms exist and use histograms. With the use of constrained inference, the approaches proposed e.g., in [3419] outperform standard methods. But they are limited to centralized contexts with a trusted third party, and only consider datasets with at most three dimensions. The PrivTree approach [41] eliminates the need of fixing the height of trees beforehand, but their security model also considers a trusted third party, and their expriments are limited to four dimensions, which is lower than the number of skills that we consider. DPBench [18] benchmarks these methods in a centralized context and considers one or two dimensions. Finally, the authors of [22] tackle the efficiency issues of privacy-preserving hierarchies of histograms. It suffers from the same dimension and privacy limitations as the above works.

Task Design. To the best of our knowledge, the problem of designing a task according to the actual crowd while providing sound privacy guarantees has not been studied by related works. Most works focus on the complexity of the task [14, on the interface with the worker [27|14|23], on the design of workflows [25|24], or on the filters that may be embedded within tasks and based on which relevant workers should be selected [3]. However, these approaches ignore the relevance of tasks with respect to the actual crowd, and thus ignore the related privacy issues.

\section{Conclusion}

We have presented a privacy-preserving approach dedicated to enabling various usages of worker profiles by the platform or by requesters, including in particular the design of tasks according to the actual distribution of skills of a population of workers. We have proposed the PKD algorithm, an algorithm resulting from rethinking the $K D$-tree construction algorithm and combining additively-homomorphic encryption with differentially-private perturbation. No trusted centralized platform is needed: the PKD algorithm is distributed between workers and the platform. We have provided formal security proofs and complexity analysis, and an extensive experimental evaluation over synthetic and realistic data that shows that the PKD algorithm can be used even with a low privacy budget and with a reasonable number of skills. Exciting future works especially include considering stronger attack models (e.g., covert or malicious adversaries), evaluating more precisely our propositions for updates, protecting the tasks in addition to worker profiles, and guaranteeing the integrity of worker profiles. 


\section{References}

1. Agrawal, R., Kiernan, J., Srikant, R., Xu, Y.: Order-preserving encryption for numeric data. In: Proc. of SIGMOD'04. pp. 563--574 (2004)

2. Aguilar-Melchor, C., Barrier, J., Fousse, L., Killijian, M.O.: Xpir: Private information retrieval for everyone. Proc. of PET'16 2016(2), 155--174 (2016)

3. Allahbakhsh, M., Benatallah, B., Ignjatovic, A., Motahari-Nezhad, H.R., Bertino, E., Dustdar, S.: Quality control in crowdsourcing systems: Issues and directions. IEEE Internet Computing 17(2), 76--81 (2013)

4. Bentley, J.L.: Multidimensional binary search trees used for associative searching. Communications of the ACM 18(9), 509--517 (1975)

5. Béziaud, L., Allard, T., Gross-Amblard, D.: Lightweight privacy-preserving task assignment in skill-aware crowdsourcing. In: Proc. of DEXA'28. pp. 18--26 (2017)

6. Boldyreva, A., Chenette, N., O'Neill, A.: Order-preserving encryption revisited: Improved security analysis and alternative solutions. In: Proc. of CRYPTO'31. pp. 578--595 (2011)

7. Chor, B., Goldreich, O., Kushilevitz, E., Sudan, M.: Private information retrieval. In: Proc. of FOCS'95. pp. 41--50 (1995)

8. Cohen, A., Nissim, K.: Linear program reconstruction in practice. CoRR (2018)

9. Cormode, G., Procopiuc, C., Srivastava, D., Shen, E., Yu, T.: Differentially private spatial decompositions. In: Proc. of ICDE'12. pp. 20--31 (2012)

10. Damgård, I., Jurik, M.: A generalisation, a simplification and some applications of paillier's probabilistic public-key system. In: Proc. of PKC'01. pp. 119--136 (2001)

11. Dinur, I., Nissim, K.: Revealing information while preserving privacy. In: Proc. of SIGACT-SIGMOD-SIGART'03. pp. 202--210 (2003)

12. Dwork, C.: Differential privacy. In: Proc. of ICALP'06. pp. 1--12 (2006)

13. Dwork, C., Roth, A.: The algorithmic foundations of differential privacy. Foundations and Trends in Theoretical Computer Science 9(3--4), 211--407 (2014)

14. Finnerty, A., Kucherbaev, P., Tranquillini, S., Convertino, G.: Keep it simple: Reward and task design in crowdsourcing. In: Proc. of SIGCHI'13. pp. 14:1--14:4 (2013)

15. Ghosh, A., Roughgarden, T., Sundararajan, M.: Universally utility-maximizing privacy mechanisms. SIAM Journal on Computing 41(6), 1673--1693 (2012)

16. Goldreich, O.: Foundations of cryptography--a primer. Foundations and Trends $\mathbb{R}$ in Theoretical Computer Science 1(1), 1--116 (2005)

17. Gupta, T., Crooks, N., Mulhern, W., Setty, S.T., Alvisi, L., Walfish, M.: Scalable and private media consumption with popcorn. In: Proc. of NSDI'16. pp. 91--107 (2016)

18. Hay, M., Machanavajjhala, A., Miklau, G., Chen, Y., Zhang, D.: Principled evaluation of differentially private algorithms using dpbench. In: Proc. of SIGMOD'16. pp. 139--154. ACM (2016)

19. Hay, M., Rastogi, V., Miklau, G., Suciu, D.: Boosting the accuracy of differentially private histograms through consistency. Proc. of the VLDB Endow. 3(1-2), 1021-$1032(2010)$

20. Kajino, H.: Privacy-Preserving Crowdsourcing. Ph.D. thesis, Univ. of Tokyo (2015)

21. Karmarkar, N., Karp, R.M.: The difierencing method of set partitioning. Tech. rep., Technical Report UCB/CSD 82/113, Computer Science Division, University of California, Berkeley (1982)

22. Kellaris, G., Papadopoulos, S., Papadias, D.: Engineering methods for differentially private histograms: Efficiency beyond utility. IEEE TKDE 31(2), 315--328 (2018) 
23. Kucherbaev, P., Daniel, F., Tranquillini, S., Marchese, M.: Crowdsourcing processes: A survey of approaches and opportunities. IEEE Internet Computing 20(2), 50--56 (2015)

24. Kulkarni, A., Can, M., Hartmann, B.: Collaboratively crowdsourcing workflows with turkomatic. In: Proc. of CSCW'12. pp. 1003--1012 (2012)

25. Kulkarni, A.P., Can, M., Hartmann, B.: Turkomatic: automatic, recursive task and workflow design for mechanical turk. In: Proc. HCOMP'11 (2011)

26. Lease, M., Hullman, J., Bigham, J.P., Bernstein, M.S., Kim, J., Lasecki, W., Bakhshi, S., Mitra, T., Miller, R.C.: Mechanical turk is not anonymous. SSRN Electronic Journal (2013)

27. Li, G., Wang, J., Zheng, Y., Franklin, M.J.: Crowdsourced data management: A survey. IEEE TKDE 28(9), 2296--2319 (2016)

28. Lu, Y., Tang, Q., Wang, G.: Zebralancer: Private and anonymous crowdsourcing system atop open blockchain. In: Proc. of ICDCS'18. pp. 853--865. IEEE (2018)

29. Mavridis, P., Gross-Amblard, D., Miklós, Z.: Using hierarchical skills for optimized task assignment in knowledge-intensive crowdsourcing. In: Proc. of WWW'16. pp. 843--853 (2016)

30. Mironov, I., Pandey, O., Reingold, O., Vadhan, S.: Computational Differential Privacy. In: Proc. of CRYPTO'29. pp. 126--142 (2009)

31. Paillier, P.: Public-key cryptosystems based on composite degree residuosity classes. In: Proc. of EUROCRYPT'99. pp. 223--238 (1999)

32. participants, F.: Imagine all the people and ai in the future of work. ACM SIGMOD blog post (2019)

33. Qardaji, W., Yang, W., Li, N.: Differentially private grids for geospatial data. In: Proc. of ICDE'13. pp. 757--768 (2013)

34. Qardaji, W., Yang, W., Li, N.: Understanding hierarchical methods for differentially private histograms. Proc. VLDB Endow. 6(14), 1954--1965 (2013)

35. Srba, I., Bielikova, M.: A comprehensive survey and classification of approaches for community question answering. ACM TWEB 10(3), 18 (2016)

36. Steutel, F.W., Van Harn, K.: Infinite divisibility of probability distributions on the real line (2003)

37. To, H., Ghinita, G., Shahabi, C.: A framework for protecting worker location privacy in spatial crowdsourcing. Proc. of the VLDB Endow. 7(10), 919--930 (2014)

38. To, H., Shahabi, C., Xiong, L.: Privacy-preserving online task assignment in spatial crowdsourcing with untrusted server. In: Proc. of ICDE'18. pp. 833--844 (2018)

39. Xia, H., Wang, Y., Huang, Y., Shah, A.: Our privacy needs to be protected at all costs: Crowd workers' privacy experiences on amazon mechanical turk. Proc. of HCI'17 1, 113 (2017)

40. Zhai, D., Sun, Y., Liu, A., Li, Z., Liu, G., Zhao, L., Zheng, K.: Towards secure and truthful task assignment in spatial crowdsourcing. World Wide Web pp. 1--24 (2019)

41. Zhang, J., Xiao, X., Xie, X.: Privtree: A differentially private algorithm for hierarchical decompositions. In: Proc. of SIGMOD'16. pp. 155--170 (2016) 